\title{
Functional MRI Responses to Passive, Active, and Observed Touch in Somatosensory and Insular Cortices of the Macaque Monkey
}

\author{
Saloni Sharma, Prosper A. Fiave, and $\odot$ Koen Nelissen \\ Laboratory for Neuro- and Psychophysiology, Department of Neurosciences, KU Leuven, 3000 Leuven, Belgium
}

\begin{abstract}
Neurophysiological data obtained in primates suggests that merely observing others' actions can modulate activity in the observer's motor cortices. In humans, it has been suggested that these multimodal vicarious responses extend well beyond the motor cortices, including somatosensory and insular brain regions, which seem to yield vicarious responses when witnessing others' actions, sensations, or emotions (Gazzola and Keysers, 2009). Despite the wealth of data with respect to shared action responses in the monkey motor system, whether the somatosensory and insular cortices also yield vicarious responses during observation of touch remains largely unknown. Using independent tactile and motor fMRI localizers, we first mapped the hand representations of two male monkeys' primary (SI) and secondary (SII) somatosensory cortices. In two subsequent visual experiments, we examined fMRI brain responses to (1) observing a conspecific's hand being touched or (2) observing a human hand grasping or mere touching an object or another human hand. Whereas functionally defined "tactile SI" and "tactile SII" showed little involvement in representing observed touch, vicarious responses for touch were found in parietal area PFG, consistent with recent observations in humans (Chan and Baker, 2015). Interestingly, a more anterior portion of SII, and posterior insular cortex, both of which responded when monkeys performed active grasping movements, also yielded visual responses during different instances of touch observation.
\end{abstract}

Key words: fMRI; grasping; macaque; motor; somatosensory; touch

Significance Statement

Common coding of one's own and others' actions, sensations, and emotions seems to be widespread in the brain. Although it is currently unclear to what extent human somatosensory cortices yield vicarious responses when observing touch, even less is known about the presence of similar vicarious responses in monkey somatosensory cortex. We therefore localized monkey somatosensory hand representations using fMRI and investigated whether these regions yield vicarious responses while observing various instances of touch. Whereas "tactile SI and SII" did not elicit responses during touch observation, a more anterior portion of SII, in addition to area PFG and posterior insular cortex, all of which responded during monkeys' own grasping movements, yielded vicarious responses during observed touch.

\section{Introduction}

The brains of social species appear endowed with mechanisms suggesting a close link between the representation of own and

Received June 7, 2017; revised Feb. 1, 2018; accepted March 3, 2018.

Author contributions: S.S. and K.N. designed research; S.S. and K.N. performed research; P.A.F. contributed unpublished reagents/analytic tools; S.S. and P.A.F. analyzed data; S.S. and K.N. wrote the paper.

This work was supported by Hercules II funds, Fonds Wetenschappelijk Onderzoek Vlaanderen (G.0.622.08, G.0.593.09), KU Leuven BOF-ZAP Startfinancing (14/10) and KU Leuven (C14/17/109). We thank W. Depuydt, M. De Paep, C. Fransen, A. Hermans, P. Kayenbergh, G. Meulemans, I. Puttemans, Dr. D. Stoianovici, C. Ulens, and S. Verstraeten for technical assistance and Dr. S. Raiguel for comments on the paper.

The authors declare no competing financial interests.

Correspondence should be addressed to Dr. Koen Nelissen, Laboratory for Neuro- and Psychophysiology, 0\&N2 Campus Gasthuisberg, KU Leuven, Herestraat 49, Bus 1021, 3000 Leuven, Belgium. E-mail: Koen.Nelissen@kuleuven.be. others' actions. Many brain regions yield common responses when an individual performs an action, and when this individual sees or hears another individual performing a similar action (Pulvermüller and Fadiga, 2010; Mooney, 2014; Rizzolatti and Sinigaglia, 2016). Electrophysiological studies examining shared responses of the monkey's own and others' actions have focused mostly on the parietofrontal motor circuit, which is involved in organizing skilled grasping movements (Kilner and Lemon, 2013; Rozzi and Coudé, 2015; Rizzolatti and Sinigaglia, 2016). Neuroimaging studies in humans on the other hand, have suggested the presence of similar shared responses that seem to extend well beyond the 
classical motor system and involve extrastriate, somatosensory, insular, cingulate, and temporal lobe cortices (Keysers et al., 2010; Molenberghs et al., 2012; Oosterhof et al., 2013).

Interestingly, in humans, vicarious responses in somatosensory and insular cortices have been found not only when observing others' actions, but also when observing others being touched, or when observing others experiencing pain or emotions. It has therefore been suggested that the somatosensory cortices might carry two types of vicarious responses or "mirror" two distinct events: on one hand, somatosensory cortices might be involved in mirroring the somatosensory aspects associated with seeing someone else performing a specific action. This type of vicarious activity is related to similar shared responses observed in the observers' motor cortices during action observation. Given that the motor and somatosensory cortices are closely linked during action execution, this somatosensory activation is thought to reflect a simulation of how it would feel to move one's own body in the same way. In addition, it has been suggested that somatosensory cortices might also mirror the somatosensory state of another individual, i.e., when observing another person being touched or in pain (for review, see Keysers et al., 2010).

To date, little is known concerning mirror-like responses in monkey somatosensory and insular cortices during action or touch observation. Although modulations during touch observation were not specifically examined, a recent electrophysiological study reported that $\sim 25 \%$ of recorded neurons in secondary somatosensory cortex and surrounding parietal operculum responded to visual stimuli, with almost one-third of these responding during observation of human goal-directed actions such as reaching, grasping, and manipulating food or objects (Hihara et al., 2015). In addition, metabolic imaging data has shown that somatosensory cortices in monkeys become recruited both when they grasp an object and when they observe another individual performing a similar action (Raos et al., 2004; Evangeliou et al., 2009).

In this study, we investigated whether the somatosensory and insular cortices in the monkey yield visual fMRI responses, particularly during observation of various examples of touch. Therefore, we first localized the hand representation in monkey primary (SI) and secondary (SII) somatosensory cortices by examining fMRI responses evoked during passive tactile stimulation of the hand. Because many neurons in somatosensory and insular regions require active motor movements to drive responses, as opposed to passive cutaneous stimulation (Robinson and Burton, 1980; Fitzgerald et al., 2004; Ishida et al., 2013), we additionally trained the monkeys to actively grasp objects in the dark in the scanner (Nelissen and Vanduffel, 2011; Nelissen et al., 2018). In a number of subsequent visual fMRI experiments, we explored the presence of visual responses to different instances of touch observation in these functionally defined SI and SII cortices and determined which aspects of the visual stimulus were instrumental in driving these responses. In Experiment 1, we investigated fMRI responses during observation of conspecific hands being touched by either a biological (human finger) or nonbiological (pliers or a brush) effectors. In Experiment 2, we investigated responses to the observation of objects being grasped or touched versus a human hand being grasped or touched.

\section{Materials and Methods}

\section{Subjects}

Two male macaque monkeys (Macaca mulatta, M1 and M2; 6-7 kg; 4-5 years of age) participated in the experiments. All animal care and experimental procedures met the national and European guidelines and were approved by the animal ethical committee of the KU Leuven. The details of the surgical procedures, training of monkeys, image acquisition, eye monitoring, and statistical analysis of monkey scans have been described previously (Vanduffel et al., 2001; Nelissen et al., 2005, 2011), and will be reviewed here briefly.

\section{Fixation training}

The monkey subjects sat in a sphinx position in a plastic monkey chair placed in a mock scanner, directly facing a liquid crystal display screen. During initial training they were required to maintain fixation within a $2 \times 2^{\circ}$ window centered on a red $\operatorname{dot}\left(0.35 \times 0.35^{\circ}\right)$ in the center of the screen. Eye position was monitored at $120 \mathrm{~Hz}$ through pupil position and corneal reflection (iScan, Illumina). During this training phase, the monkeys were rewarded (fruit juice) for fixating the small red dot within the fixation window for long periods (up to several minutes).

\section{Tactile localizer}

To functionally localize tactile hand representations in somatosensory cortex, we performed a tactile localizer experiment in the scanner (Fig. $1 A$ ). To avoid visual or motor confounds during passive tactile stimulation, subjects were lightly sedated using ketamine (dosage: $12 \mathrm{mg} / \mathrm{kg} / \mathrm{h}$ ) throughout the scan, while keeping the eyes covered. As in Chan and Baker (2015), an experimenter in the scanner room brushed the subject's face or hand at the rate of $2 \mathrm{~Hz}$, using a small brush. Subject's head and arms were immobilized during the scans to avoid movements. A visual cue was presented on a small screen in the scanner in order for the experimenter to keep track of the exact timing during the stimulation blocks. During the scan, the experimenter delivered gentle brush strokes to either (1) the left or (2) right side of the subject's face (moving in a downward motion from below the eye toward the corner of the mouth), or to (3) the left or (4) right palm of the hand (starting from the center of the palm toward the fingertips). It should be noted that our localization of "tactile SI and SII" was based upon cutaneous stimulation while monkeys were lightly sedated with ketamine. Although our results are in line with other nonhuman primate fMRI studies showing robust fMRI activations can be detected in SI and SII cortices under light anesthesia throughout the somatosensory system during nociceptive or tactile processing (Chen et al., 2012; Wu et al., 2017), it is possible that passive cutaneous stimulation when monkey subjects were awake, might have resulted in more extensive or additional fMRI activations throughout the somatosensory cortices.

\section{Motor localizer}

Subjects were trained to grasp three spheres of different sizes $(16,23$, or $40 \mathrm{~mm}$ radius; Fig. $1 B$ ) with the right hand. The grasping task was previously described in detail (Nelissen and Vanduffel, 2011; Nelissen et al., 2018) and will be summarized briefly. During fixation baseline condition, monkeys were rewarded for fixating on a red fixation point (Fig. 1B) while keeping their hand in the start position. A grasping trial started when the monkey placed his hand in the start position and fixated on a green fixation point displayed centrally on the screen in front of him. After a random fixation time $(500-1500 \mathrm{~ms})$, the green fixation point changed to blue, indicating to the monkey that he could now reach and grasp the object with the right hand. After the monkey had grasped the object, he was required to lift it $5 \mathrm{~mm}$ and hold it in that position for at least $500 \mathrm{~ms}$ (maximum holding time $2000 \mathrm{~ms}$ ) to receive a juice reward. After delivery of the reward, a new trial started (green fixation point) as soon as the monkey returned his hand to the initial starting position while keeping fixation. Optic fiber cables were positioned at the start and end positions of the hand's reaching trajectory, as well as at three locations along the hand/arm path, allowing us to track the location of the hand and arm and to time the execution of the motor tasks (Nelissen and Vanduffel, 2011; Nelissen et al., 2018). As a control, the monkeys also performed a reach-only task without grasping any object. For this motor task, the disk was positioned so that the empty slot (not containing an object) was positioned in front of the monkey. The monkey was required to reach forward and place his hand onto this empty slot on the disk. The visual stimuli used to cue the monkey and the timing parameters were exactly as during the reach-and-grasp task. After reaching, the monkey was required to rest his open hand on the disk for at least $500 \mathrm{~ms}$ to 


\section{A Passive Touch Localiser}

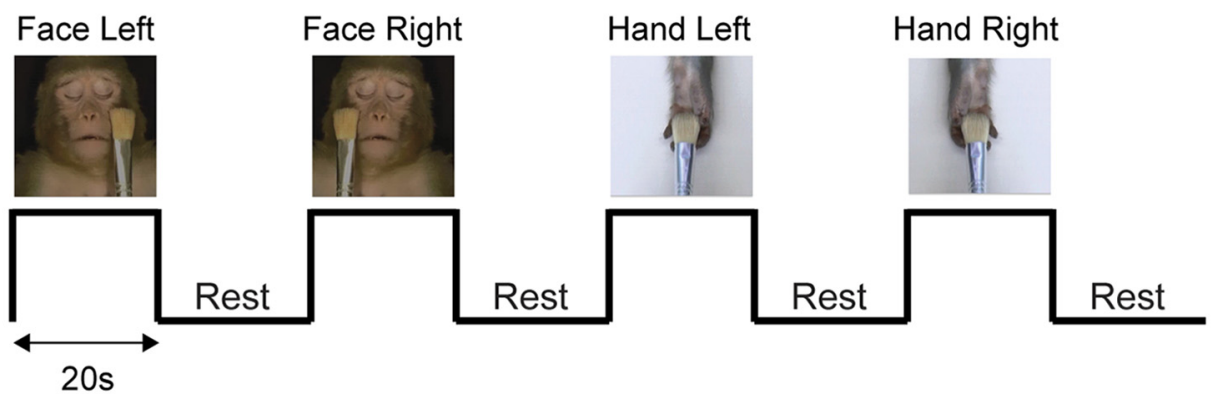

\section{B Active Touch Localiser}

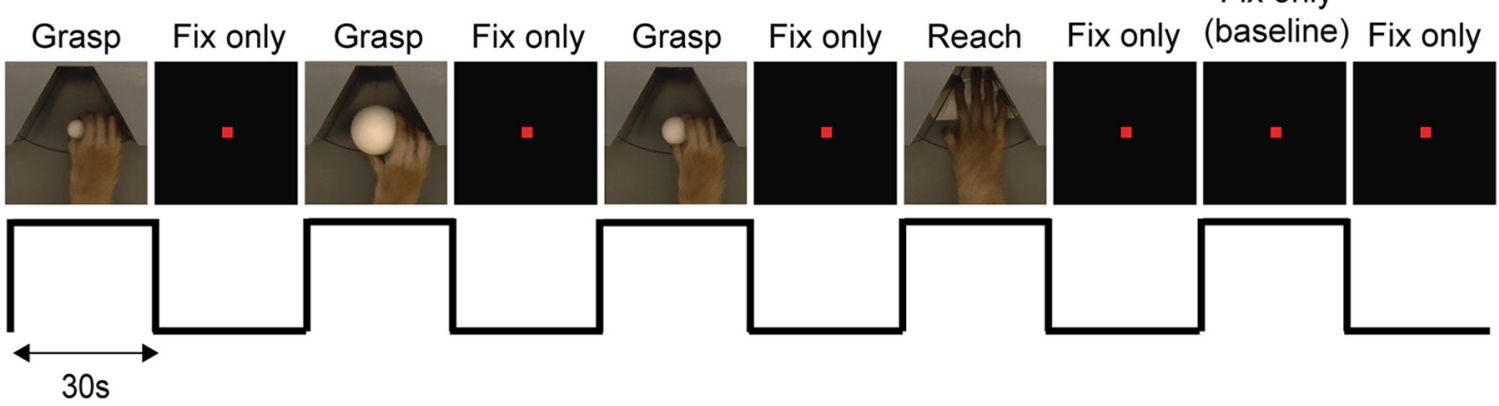

\section{Visual Experiment 1}
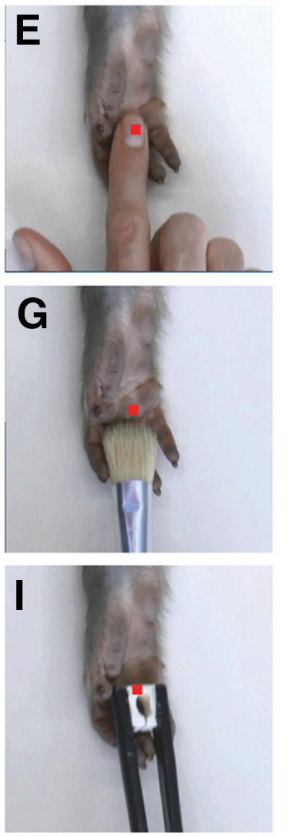

Experiment 1

E Hand Touch

F Control Hand Touch

G Brush Touch

H Control Brush Touch

I Plier Touch

J Control Plier Touch
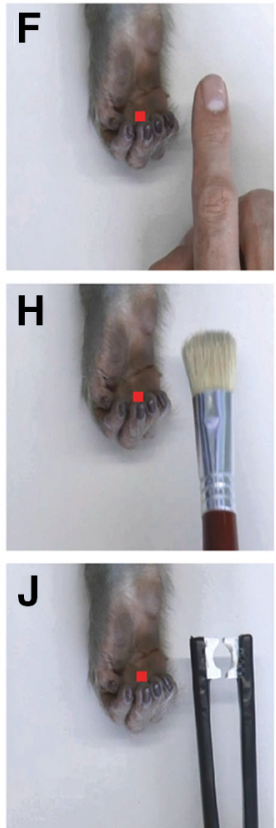

\section{Experiment 2}

K Grasp Object

L Control Grasp Object

M Touch Object

N Control Touch Object

O Grasp Hand

P Control Grasp Hand

Q Touch Hand

R Control Touch Hand
D Visual Experiment 2
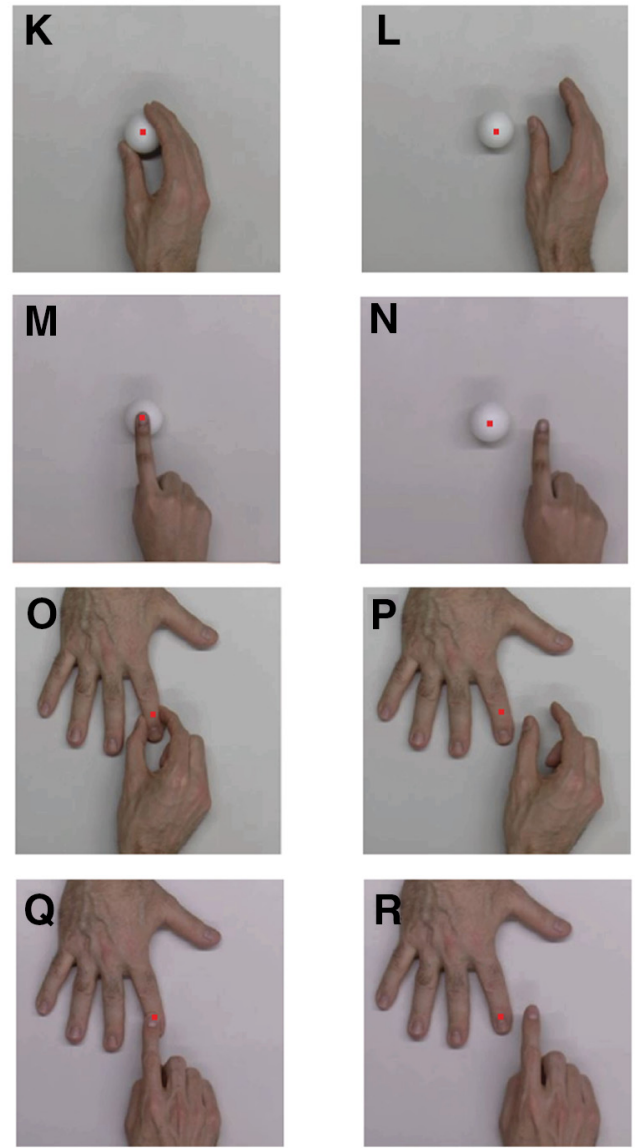

Figure 1. Functional MRI experiments and stimuli. $\boldsymbol{A}$, To localize the hand representations in SI and SII responsive to passive cutaneous stimulation, we brushed the subject's left or right palm or side of the face, under light sedation in the scanner. Cutaneous stimulation blocks were interleaved with no stimulation (rest) blocks of equal duration (20 s). $\boldsymbol{B}$, In an active touch localizer, subjects either grasped spheres of different diameters (Grasp) or placed their open right hands on a disk in front of them (Reach) in the dark inside the scanner. Motor blocks ( $30 \mathrm{~s}$ ) were interleaved with baseline fixation blocks (Fix only), during which subjects were rewarded for fixating a red fixation target. C, In visual Experiment 1, we examined fMRI responses to observation of a conspecific's hand being touched by different effectors. Monkeys were required to fixate a small red fixation spot positioned on top of a monkey hand, while either a human hand (E), a brush ( $\boldsymbol{G}$ ), or a pair of closed pliers (I) touched the monkey's palm and swept down toward the fingertips. As controls, videos depicted the same effectors making a similar translation movement (Figure legend continues.) 
Table 1. Results of repeated-measures two-way ANOVA and post hoc tests for Experiment 1

\begin{tabular}{|c|c|c|c|c|c|}
\hline & \multicolumn{2}{|l|}{ Insula } & \multicolumn{2}{|l|}{ Visual SII } & \multirow[b]{2}{*}{ PFG right } \\
\hline & Left & Right & Left & Right & \\
\hline \multicolumn{6}{|l|}{ Main effect of touch (touch vs no touch) } \\
\hline \multicolumn{6}{|l|}{ Monkey M1 } \\
\hline$F_{(1,31)}$ & 32.89 & 27.28 & 8.926 & 12.21 & 31.78 \\
\hline$p^{(1,3)}$ & $3 \times 10^{-5}$ & $1 \times 10^{-5}$ & 0.005 & 0.0015 & $3 \times 10^{-6}$ \\
\hline \multicolumn{6}{|l|}{ Monkey M2 } \\
\hline$F_{(1,29)}$ & 71.01 & 77.09 & 115.4 & 226.5 & 79.83 \\
\hline$p^{p}$ & $2.89 \times 10^{-9}$ & $1.2 \times 10^{-9}$ & $1.3 \times 10^{-11}$ & $3 \times 10^{-15}$ & $7.96 \times 10^{-10}$ \\
\hline \multicolumn{6}{|l|}{ Bonferroni post hoc tests } \\
\hline \multicolumn{6}{|l|}{ Monkey M1 } \\
\hline Hand touch vs control hand touch & 0.00353 & 0.0062 & 0.202 & 0.517 & 0.0018 \\
\hline Brush touch vs control brush touch & 0.118 & 0.0013 & 0.525 & 0.019 & 0.357 \\
\hline Plier touch vs control plier touch & 0.0034 & 0.026 & 0.045 & 0.024 & 0.0001 \\
\hline \multicolumn{6}{|l|}{ Monkey M2 } \\
\hline Hand touch vs control hand touch & $8.40 \times 10^{-5}$ & $6.80 \times 10^{-9}$ & $3.10 \times 10^{-8}$ & $5 \times 10^{-11}$ & $1.39 \times 10^{-10}$ \\
\hline Brush touch vs control brush touch & $1.60 \times 10^{-6}$ & $2.60 \times 10^{-5}$ & $2.89 \times 10^{-7}$ & $4.58 \times 10^{-8}$ & 0.0093 \\
\hline Plier touch vs control plier touch & $7.70 \times 10^{-5}$ & $1.20 \times 10^{-5}$ & $1.7 \times 10^{-8}$ & $1 \times 10^{-11}$ & $2.55 \times 10^{-7}$ \\
\hline
\end{tabular}

receive the juice reward. By using a subtraction technique, this control task allowed us to isolate those brain regions specifically activated during haptic touch and grasp of an object (Nelissen and Vanduffel, 2011; Nelissen et al., 2018).

\section{Visual experiments}

During the visual experiments, monkeys were rewarded with drops of apple juice for fixating on a small red dot displayed in the center of the screen while different videos were presented.

Experiment 1. In Experiment 1 (Fig. 1C), visual stimuli consisted of video clips displaying a conspecific hand being touched by 3 different effectors: either a human hand (Fig. 1E), a brush (Fig. $1 G$ ), or a pair of pliers (Fig. 1I) touched a monkey hand near the center of the palm and swept downward toward the fingers. As controls for each of these videos depicting touch, we used videos showing the same three effectors (human hand, brush, pliers) making similar translation movements next to the monkey hand, without touching it (Fig. $1 F, H, J$ ). To add variation to the videos, all the original videos, which depicted a right human hand (or a right hand holding the brush or pliers) were also flipped along the vertical axis to create a second set of action videos. All videos of Experiment 1 measured $13 \times 11.5$ visual degrees, and had a duration of $4 \mathrm{~s}$.

Experiment 2. In Experiment 2 (Fig. 1D), visual stimuli consisted of video clips depicting four different types of actions depicting touch: a human hand grasping either a sphere (Fig. $1 K$ ) or a finger (Fig. 1O), and a human hand merely touching a sphere (Fig. 1M) or a finger (Fig. 1Q). The "human hand grasping a sphere" and "human hand touching a sphere" conditions consisted of six examples each, depicting three different-sized spheres (16, 23, and $40 \mathrm{~mm}$ diameter) being grasped (or touched) by a male hand and a female hand. The same objects were also used in the active grasping localizer during which monkeys grasped these objects in the dark (see above). For the "human hand grasping a finger" and the "human hand touching finger" conditions, three different hands (1 male and 2 female) were grasped (or touched) by the same male and female hand as in the grasp videos, again resulting in six different examples for each of these conditions. As controls for each of these actions depicting touch, we also used videos that showed the same human hands making similar translational movements without touching the sphere or finger (Fig. $1 L, N, P, R$ ). As in Experiment 1, all original videos, depicting the right hand of a human actor performing the various actions, were also

$\leftarrow$

(Figure legend continued.) next to the monkey hand without touching it $(\boldsymbol{F}, \boldsymbol{H}, \boldsymbol{J})$. $\boldsymbol{D}$, In visual Experiment 2, we investigated fMRI responses to observing grasping and touching. Videos included an object being grasped $(\boldsymbol{K})$ or touched with a finger $(\boldsymbol{M})$, in addition to a human hand being grasped ( $\mathbf{O}$ ) or touched with a finger $(\mathbf{Q})$. As controls, videos depicted the same hands making a similar translational movement next to the object or hand, without touching them $(\boldsymbol{L}, \boldsymbol{N}, \boldsymbol{P}, \boldsymbol{R})$. flipped along the vertical axis. The videos used in Experiment 2 measured $10 \times 10$ visual degrees and were $4 \mathrm{~s}$ in duration.

\section{Experimental design and statistical analysis}

Scanning. Functional images were acquired with a 3.0 tesla full-body scanner (Siemens, MAGNETOM Prisma ${ }^{\text {fit }}$ ), using a gradient-echo T2* weighted echoplanar imaging sequence ( 40 horizontal slices; TR: $2 \mathrm{~s}$; TE: $17 \mathrm{~ms} ; 1.25 \times 1.25 \times 1.2 \mathrm{~mm}^{3}$ voxels) with a custom eight-channel phased-array receive coil, and a saddle-shaped, radial transmit-only surface coil (Kolster et al., 2009).

Before each scanning session, a contrast agent, monocrystalline iron oxide nanoparticle (MION; Molday ION, BioPAL), was injected into the femoral/saphenous vein $(6-11 \mathrm{mg} / \mathrm{kg})$ of the monkey. Use of the contrast agent improved the contrast-noise ratio by approximately fivefold (Vanduffel et al., 2001) and enhanced spatial selectivity of the magnetic resonance (MR) signal changes (Zhao et al., 2006), compared with blood oxygenation level-dependent (BOLD) measurements. Whereas BOLD measurements depend on cerebral blood volume (CBV), blood flow, and oxygen extraction, MION measurements depend only on blood volume (Mandeville et al., 1999). Accordingly, we have inverted the polarity of all signal-change values to account for the difference between MION CBV and BOLD activation maps (increased brain activation produces a decrease in MR signal in MION CBV maps).

For the tactile localizer, a block design was used consisting of five conditions: brush left side or right side of face, brush left or right palm of the hand, and no brush (rest) baseline (Fig. 1A). A full run consisted of 5 start volumes, followed by the four stimulation blocks, all of which lasted $20 \mathrm{~s}$ (10 volumes), with equally long rest blocks between the conditions, by this sequence of blocks was repeated once in the same run. The total run length consisted of 165 volumes. For Monkeys M1 and M2, respectively, 36 runs and 18 runs were collected. For the tactile localizer group analysis, data from all these runs were combined $(n=54)$. The motor localizer consisted of a block design (Fig. $1 B$ ) with alternating 15 volume (30 s) blocks of motor tasks and fixation baseline. A typical run consisted of five start volumes followed by the following sequence of blocks $(30 \mathrm{~s}$ grasp sphere $16 \mathrm{~mm}-30 \mathrm{~s}$ fix only-30 s grasp sphere $40 \mathrm{~mm}-30 \mathrm{~s}$ fix only-30 s grasp sphere $23 \mathrm{~mm}-30 \mathrm{~s}$ fix only-30 s reach-30 s fix only-30 $\mathrm{s}$ fixation baseline $-30 \mathrm{~s}$ fix only), repeated once in the same run. A full run thus consisted of 305 volumes. For Monkey M2, the same design was used, except that the objects to be grasped were presented in a different order within the runs $(23 \mathrm{~mm}$ sphere, $16 \mathrm{~mm}, 40 \mathrm{~mm}$ ) compared with Monkey M1. For Monkey M1, 11 runs were collected with one run discarded due to poor performance. For Monkey 2, 12 runs were collected. For the group data analysis, all grasping trials from all these runs were combined $(n=22)$. For visual Experiment 1 (Fig. $1 C$ ), a block design was used with seven conditions: hand touch hand, control touch hand, brush touch hand, control brush touch, pliers touch hand, control pliers touch (Fig. $1 E-J$ ), and fixation-only baseline. Different example videos belong- 
Table 2. Results of repeated-measures two-way ANOVA and post hoc tests for object actions from Experiment 2

\begin{tabular}{|c|c|c|c|c|c|c|}
\hline & \multicolumn{2}{|l|}{ Insula } & \multicolumn{2}{|l|}{ Visual SII } & \multicolumn{2}{|l|}{ PFG } \\
\hline & Left & Right & Left & Right & Left & Right \\
\hline \multicolumn{7}{|l|}{ Main effect of touch (touch vs no touch) } \\
\hline \multicolumn{7}{|l|}{ Monkey M1 } \\
\hline$F_{(1,41)}$ & - & 5.07 & 6.946 & 12.25 & - & 8.402 \\
\hline$p$ & - & 0.03 & 0.012 & 0.0011 & - & 0.006 \\
\hline \multicolumn{7}{|l|}{ Monkey M2 } \\
\hline$F_{(1,41)}$ & 3.435 & 19.97 & 7.97 & 13.66 & 0.655 & 5.72 \\
\hline$p^{(1,41)}$ & 0.071 & $6.1 \times 10^{-5}$ & 0.0073 & 0.0006 & 0.432 & 0.022 \\
\hline \multicolumn{7}{|l|}{ Bonferroni post hoc tests } \\
\hline \multicolumn{7}{|l|}{ Monkey M1 } \\
\hline Grasp object vs control grasp object & - & 0.64 & 0.0112 & 0.0009 & - & 0.052 \\
\hline Touch object vs control touch object & - & 0.0067 & $>0.99$ & 0.33 & - & 0.74 \\
\hline \multicolumn{7}{|l|}{ Monkey M2 } \\
\hline Grasp object vs control grasp object & 0.303 & 0.102 & 0.22 & 0.0021 & $>0.99$ & 0.0066 \\
\hline Touch object vs control touch object & 0.41 & 0.00016 & 0.42 & 0.022 & $>0.99$ & $>0.99$ \\
\hline
\end{tabular}

Table 3. Results of repeated-measures two-way ANOVA and post hoc tests for hand actions from Experiment 2

\begin{tabular}{|c|c|c|c|c|c|c|}
\hline & \multicolumn{2}{|l|}{ Insula } & \multicolumn{2}{|l|}{ Visual SII } & \multicolumn{2}{|l|}{ PFG } \\
\hline & Left & Right & Left & Right & Left & Right \\
\hline \multicolumn{7}{|l|}{ Main effect of touch (touch vs no touch) } \\
\hline \multicolumn{7}{|l|}{ Monkey M1 } \\
\hline$F_{(1,41)}$ & - & 1.12 & 0.82 & 8.043 & - & 5.914 \\
\hline$p^{(1,+1)}$ & - & 0.30 & 0.37 & 0.0072 & - & 0.019 \\
\hline \multicolumn{7}{|l|}{ Monkey M2 } \\
\hline$F_{(1,41)}$ & 22.8 & 6.04 & 28.14 & 17.85 & 17.92 & 53.3 \\
\hline$p^{(1,41)}$ & $2.3 \times 10^{-5}$ & 0.018 & $4 \times 10^{-6}$ & 0.0001 & 0.0001 & $6.2 \times 10^{-9}$ \\
\hline \multicolumn{7}{|l|}{ Bonferroni post hoc tests } \\
\hline \multicolumn{7}{|l|}{ Monkey M1 } \\
\hline Grasp hand vs control grasp hand & - & $>0.99$ & 0.076 & 0.026 & - & 0.046 \\
\hline Touch hand vs control touch hand & - & 0.044 & 0.91 & 0.71 & - & 0.086 \\
\hline \multicolumn{7}{|l|}{ Monkey M2 } \\
\hline Grasp hand vs control grasp hand & 0.0012 & 0.25 & 0.0002 & 0.0003 & $6 \times 10^{-5}$ & $1.99 \times 10^{-8}$ \\
\hline Touch hand vs control touch hand & 0.76 & 0.23 & 0.41 & 0.098 & 0.048 & 0.015 \\
\hline
\end{tabular}

ing to the same condition were presented randomly within the same block. Each block (condition) lasted for 10 volumes (20 s) and was presented thrice in one run. Each run thus included 215 volumes $(7 \mathrm{~min}$, 16 s). For single-subject data analysis, 32 runs from Monkey M1 and 30 runs from Monkey M2 were used. Group analysis consisted of these combined 62 runs. For visual Experiment 2 (Fig. 1D), a block design was used that contained nine conditions: grasp object, control grasp object, grasp hand, control grasp hand, touch object, control touch object, touch hand, control touch hand (Fig. $1 K-R$ ), and fixation-only baseline. Each block (condition) lasted for 10 volumes $(20 \mathrm{~s})$ and was presented twice in one run. Each run consisted of 185 volumes ( 6 min, 16 s). For singlesubject data analysis, 42 runs were used from Monkey M1 and 42 runs from Monkey M2. Group analysis consisted of these combined 84 runs.

Data preprocessing and GLM fitting. Data were preprocessed and analyzed using statistical parametric mapping (SPM5; RRID:SCR_007037) software and JIP (JIP Analysis Toolkit v2.x; RRID:SCR_009588). Spatial preprocessing consisted of realignment and nonrigid coregistration (using JIP) of the functional images to a high-resolution anatomical template of the monkey's own brain. For displaying group data, the functional data of Monkey M1 were also warped to Monkey M2's anatomical brain template. Functional volumes were then resliced to $1 \mathrm{~mm}^{3}$ isotropic and smoothed with a $1.5 \mathrm{~mm}$ (FWHM) Gaussian kernel. Condition MION response amplitude at each voxel was estimated using a general linear model (GLM) following previously detailed procedures for fitting GLM (Friston et al., 1994; Vanduffel et al., 2001). To do this, a MION hemodynamic response function was convolved with a boxcar model representing the various stimulus conditions (Vanduffel et al., 2001). The influence of head motion was accounted for by including six regressors of no interest in the GLM model corresponding to three rotations and translations along $x$-, $y$-, and $z$-axes. In addition, horizontal and vertical components of the eye movement traces were also included in the GLM as regressors of no interest to remove eye-movement-related confounds. For each run, a GLM was fitted for each voxel resulting in a map ( $\beta$ map) for each condition of interest and for the eight regressors of no interest.

Univariate whole-brain analysis. Both fixed-effect group (allowing inference only at the group level) and single-subject data are presented. For single-subject analyses, functional images are warped onto the monkey's own anatomy. For the group analyses, functional data of Monkey M1 was warped to the anatomical template of Monkey M2. For display purposes, whole-brain group SPM T-maps are rendered on an inflated brain template of Monkey M2 using FreeSurfer toolbox (FreeSurfer v5.3.0; RRID: SCR_001847). In addition, single-subject SPM T-maps are represented on coronal sections of each monkey's individual anatomical template. Significance was set at $p<0.05$ corrected (familywise error) for multiple comparisons at voxel level or at $p<0.001$, uncorrected.

Delineation of tactile SI and SII regions-of-interest. To delineate a functional regions-of-interest (ROIs) for SI and SII in each hemisphere of a given monkey, we contrasted hand stimulation versus ipsilateral face stimulation in our tactile localizer data. In both subjects, our tactile localizer experiment yielded significant contralateral responses during stimulation of the hand in a portion of the postcentral sulcus and adjacent crown. Since it is difficult to draw exact borders of the different subregions of SI (areas 3a, 3b, 1, and 2), we refer to our functional activation as tactile SI throughout the paper. Inspection of the local maxima of this activation on a high-resolution anatomical template of each subject suggests this functional activation was centered around the posterior bank of the central sulcus and adjacent crown, extending toward the intraparietal sulcus (corresponding to areas 3b, 1, and 2). Our functionally defined tactile SI ROIs consisted of 84 and 115 voxels for left 


\section{Passive touch}

\section{A Hand Right vs Face Right}

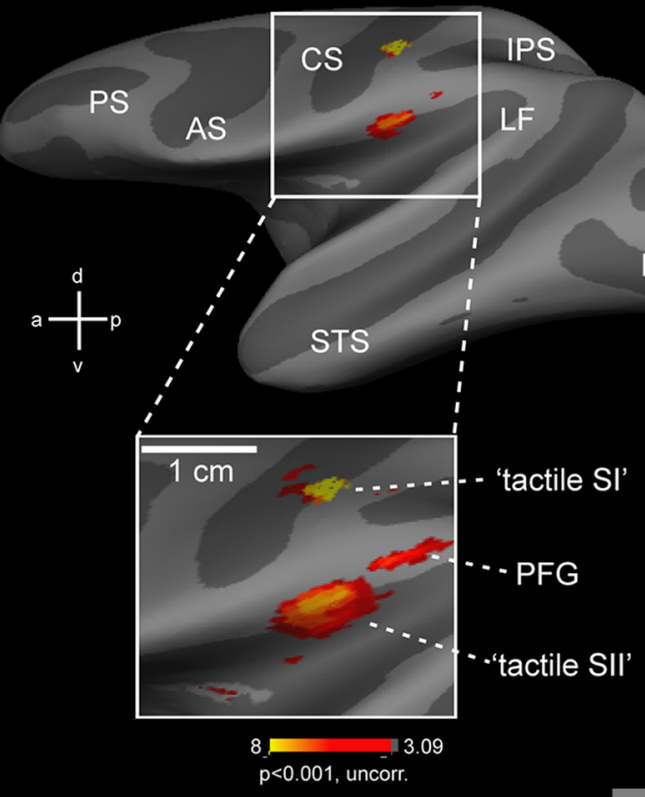

\section{Active touch}

Grasp vs Reach (contralateral)

C

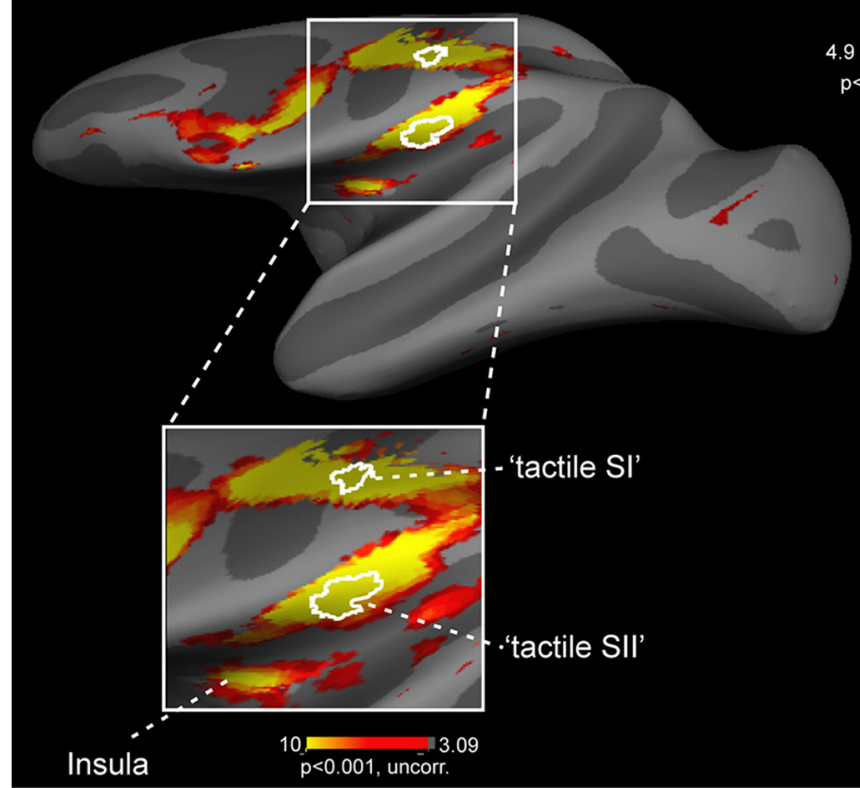

B

\section{Hand Left vs Face Left}

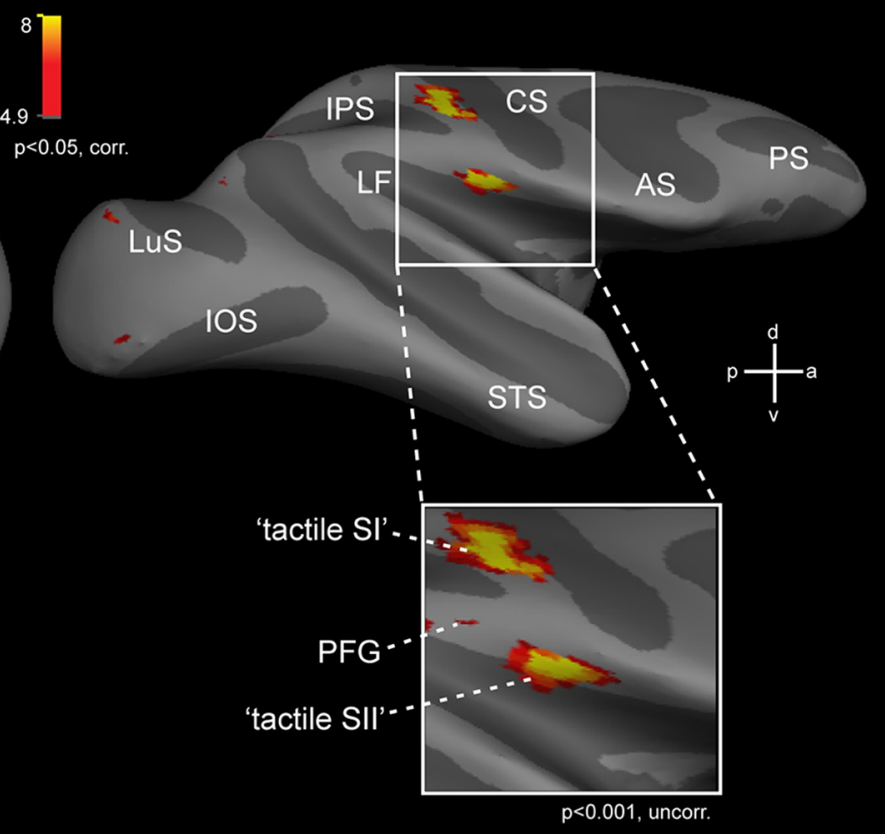

D

Grasp vs Reach (ipsilateral)

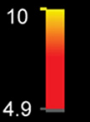

$p<0.05$, corr
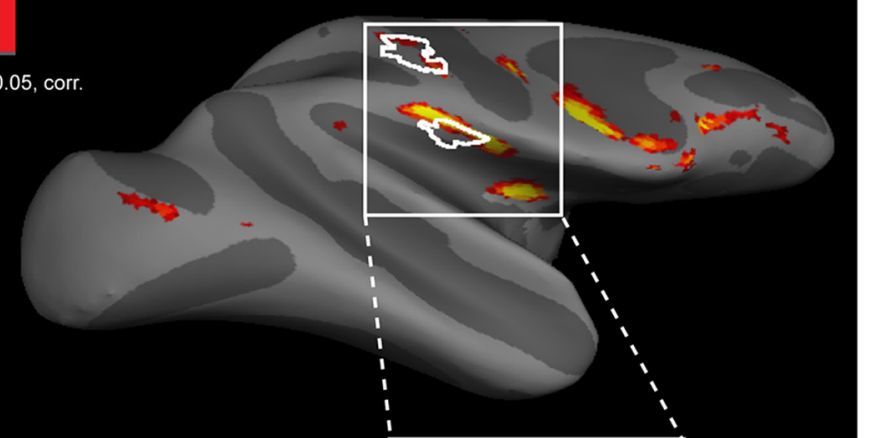

'tactile SI'-

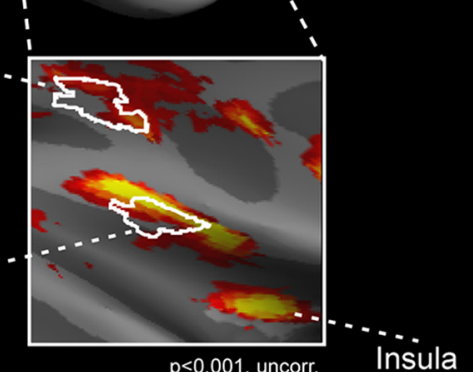

'tactile SII'- -

$p<0.001$, uncorr.

Figure 2. Whole-brain fMRI responses during passive and active touch. $A, B$, Cutaneous stimulation of the monkeys' hands (compared with stimulation of the ipsilateral side of the face) yielded significant ( $p<0.05$, corr) fMRI responses in contralateral SI (tactile SI) and SII (tactile SII). Insets indicate fMRI responses for the same contrast at $p<0,001$, uncorr. $C$, Active touch during grasp execution (compared with reach-only) in the dark yielded significant $(p<0.05$, corr) contralateral responses in early visual, parietal, motor, premotor, prefrontal, somatosensory, and insular regions. White outlines indicate locations of tactile SI and SII regions responding during passive cutaneous stimulation in $\boldsymbol{A}$ and $\boldsymbol{B}$. Insets, fMRI responses for the same contrast at $p<0,001$, uncorr. $\boldsymbol{D}$, In the ipsilateral hemisphere, grasping (compared with reach-only) yielded more restricted fMRI responses in similar early visual, parietal, motor, premotor, prefrontal, somatosensory, and insular regions. 


\section{Visual Experiment 1}

\section{A All touch vs controls}

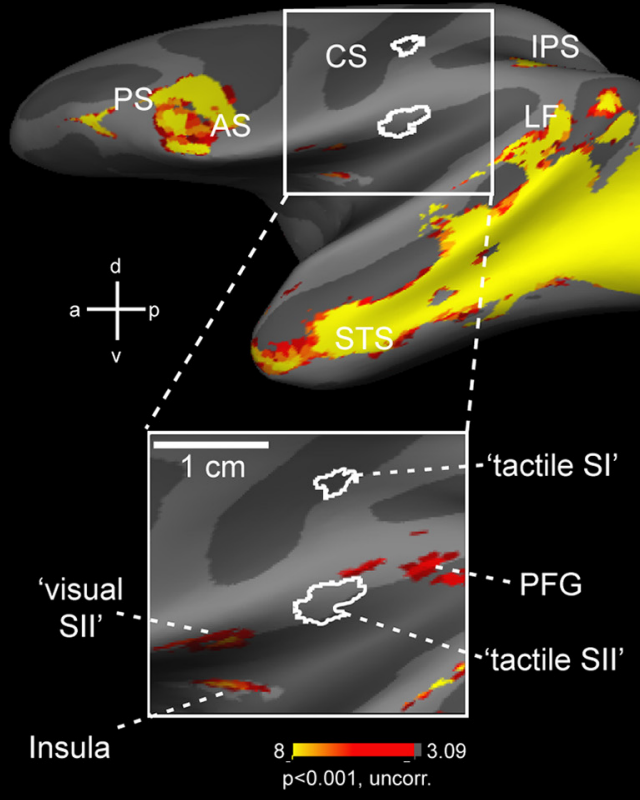

\section{Visual Experiment 2}

C All touch vs controls

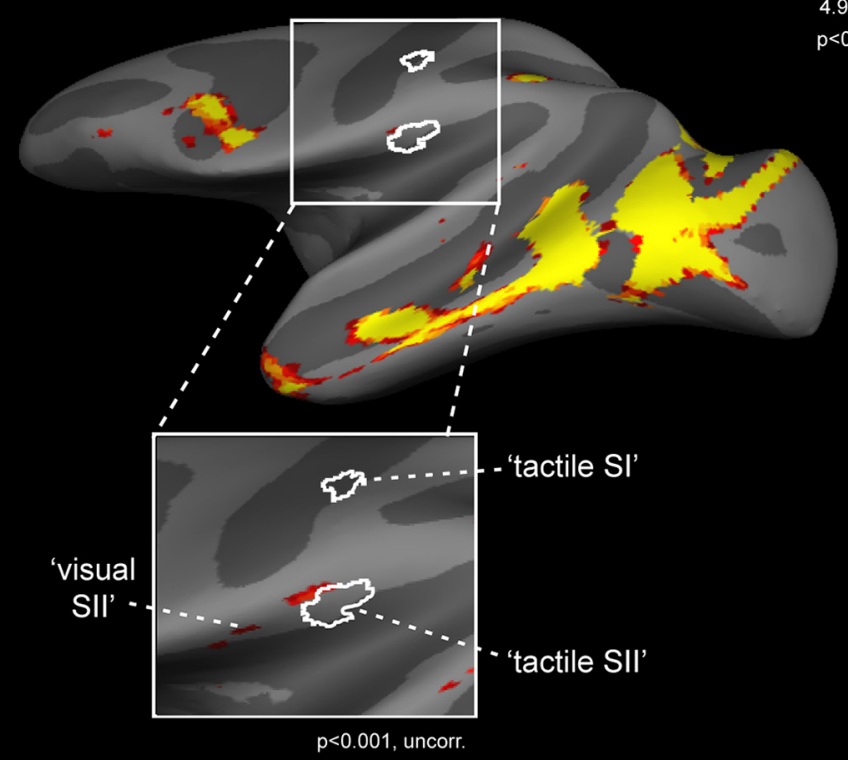

B All touch vs controls

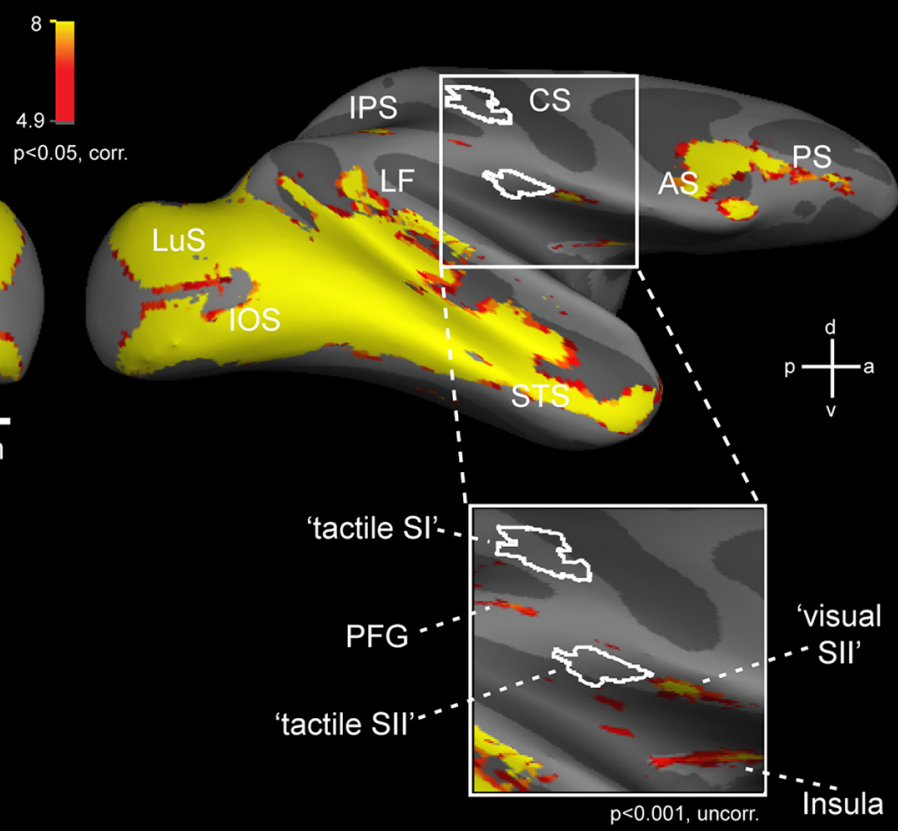

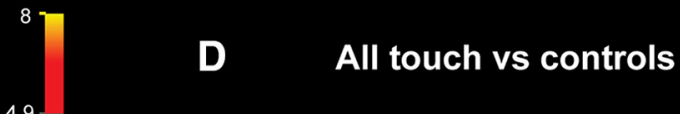

$\mathrm{p}<0.05$, corr.

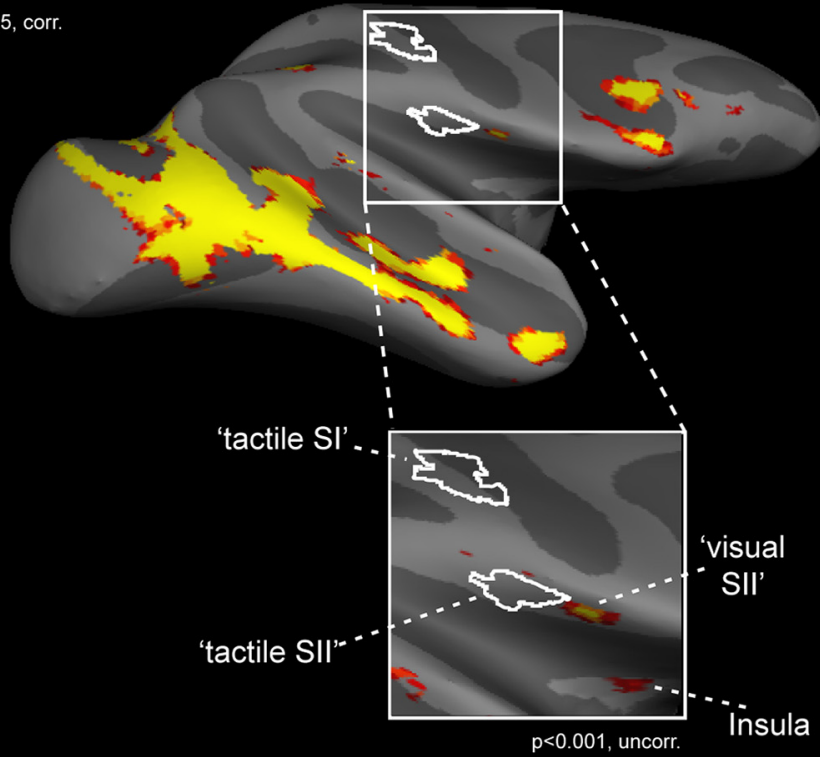

Figure 3. Whole-brain fMRI responses during observation of touch. $\boldsymbol{A}, \boldsymbol{B}$, Observing a conspecific's hand being touched with different effectors (vs no-touch controls) yielded significant ( $p<$ 0.05 , corr) responses in early visual, extrastriate, superior temporal sulcus (STS), parietal, premotor, prefrontal, somatosensory, and insular regions. Tactile SI and SII (white outlines) did not elicit significant responses to observation of a conspecific's hand being touched. Lowering the statistical threshold ( $p<0.001$, uncorr) confirmed the absence of touch-observation related responses (insets) in tactile SI and SII (white outlines). C, D, Observing an object or human hand being grasped or touched (vs no-touch controls) yielded significant ( $p<0.05$, corr) responses in similar early visual, extrastriate, STS, parietal, premotor, prefrontal, somatosensory, and insular regions. As for visual Experiment 1, tactile SI and tactile SII (white outlines) did not produce significant responses to observation of various instances of touch (vs controls). Lowering the statistical threshold ( $p<0.001$, uncorr) confirmed the absence of touch observation related responses (insets) in tactile SI and SII (white outlines). 

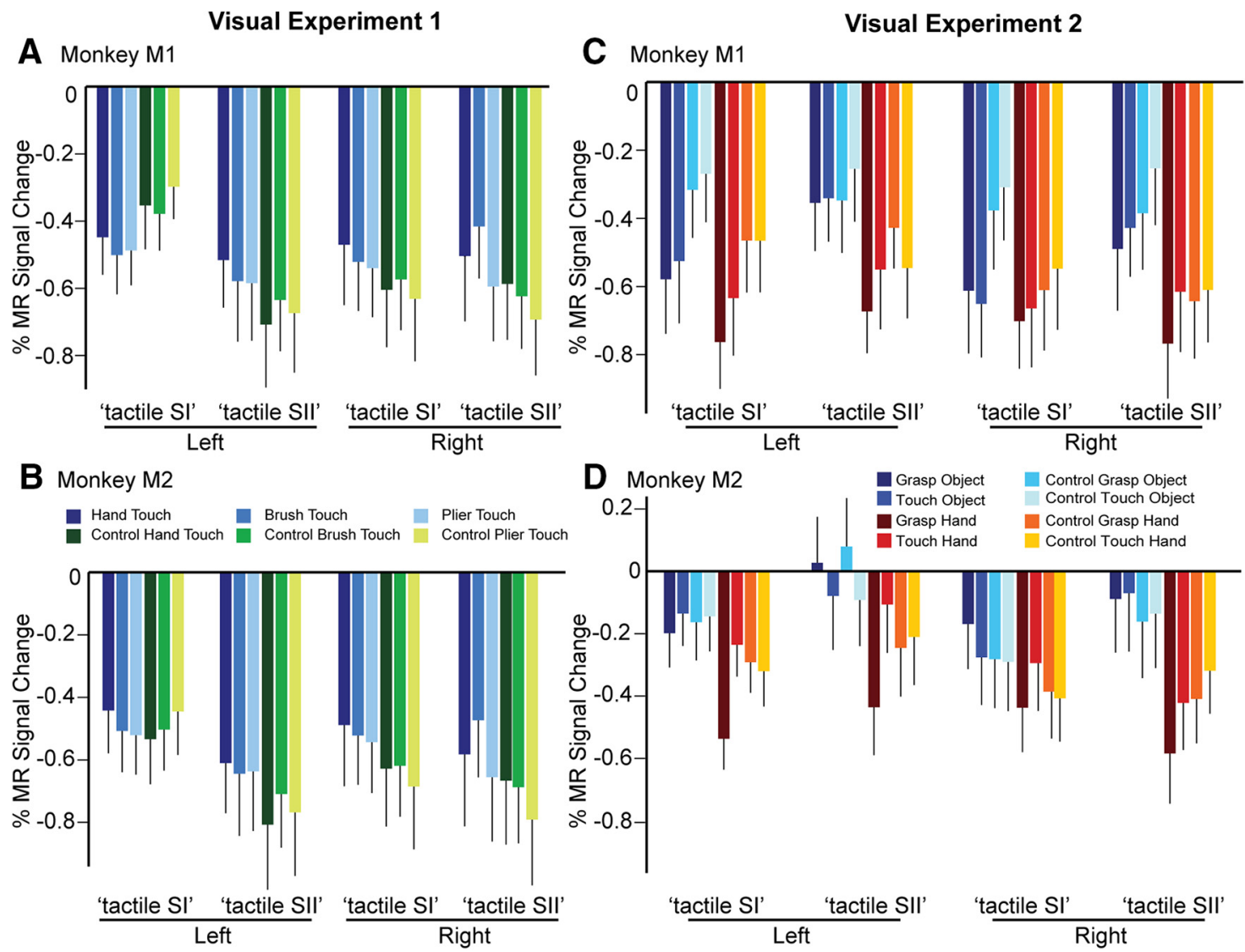

Figure 4. Univariate fMRI responses to observed touch in tactile SI and SII regions-of-interest. $A, B$, Observing a conspecific's hand being touched by a human hand, brush or pair of pliers, in addition to no-touch control videos, did not yield significant visual responses (compared with fixation-only baseline) in tactile SI or SII in either hemisphere in any of the two monkey subjects ( $\boldsymbol{A}$, Monkey M1; $\boldsymbol{B}$, Monkey M2). C, D, Observing a human hand grasping or merely touching either an object or another human hand, in addition to no-touch control videos, elicited no significant visual responses (compared with fixation-only baseline) in tactile SI or SIl in either hemisphere in any of the two monkey subjects (C, Monkey M1; D, Monkey M2).

and right hemispheres in Monkey M1 (contralateral hand vs face stimulation, $p<0.001$, uncorr), and 205 and 149 voxels for left and right hemispheres in Monkey M2 (contralateral hand vs face stimulation, $p<$ 0.05 , corr).

In addition to SI, contrasting hand versus ipsilateral face stimulation also yielded a consistent contralateral activation in the upper bank of the lateral sulcus, where SII has been described. Because there currently is no general agreement with respect to the exact extent or the possible clustering of functional properties into different subfields of SII and surrounding cortex, throughout the paper we refer to the portion of the lateral sulcus that yielded significant responses during passive cutaneous stimulation of the hand palm and fingers, as tactile SII. The region in upper bank of the lateral sulcus anterior to this area, where visual responses for actions and touch were observed in our visual experiments, we refer to as visual SII. Our functionally defined tactile SII ROIs consisted of 22 and 36 voxels for left and right hemispheres in Monkey M1 (contralateral hand vs face stimulation, $p<0.001$, uncorr), and 27 and 55 voxels for left and right hemispheres in Monkey M2 (contralateral hand vs face stimulation, $p<0.05$, corr).

Univariate ROI-based analysis. ROI-based analysis of visual responses (Experiments 1 and 2) in functionally defined tactile SI and SII regions was performed using MarsBar (MarsBaR region-of-interest toolbox for SPM v0.41; RRID:SCR_009605). Percentage signal changes (mean from all voxels in these ROIs) for the different visual conditions in Experiments 1 and 2 were plotted against fixation baseline (see Fig. 4). For the visual responses shown in Figures $6-11$, the percentage signal change for every condition (relative to the fixation baseline) at the local maxima was extracted for each run. We then assessed significance using repeatedmeasures factorial ANOVA, followed by a Bonferroni post hoc test correcting for the number of comparisons in the test. For Experiment 1, we conducted a two-way repeated-measures ANOVA with the main effect of touch versus no touch, and the different effectors (human hand, brush, pliers) as factors (Table 1). In addition to examining the main effect of touch (all effectors vs all controls), we performed post hoc Bonferroni tests to investigate each effector versus its controls and possible differences between effectors (Table 1). For Experiment 2, we split the analysis into two separate $2 \times 2$ factorial designs. In the first factorial design we considered only the conditions with the object present (Fig. $1 K-N$ ). In the second factorial design, we considered only the conditions with the static hand present (Fig. 1O-R). We performed two repeated-measures two-way ANOVAs with the main effect of touch versus no touch as one factor and grasps versus touches as the second factor, followed by post hoc Bonferroni tests to test whether grasps or touches were significantly different from one another and from their respective controls (Tables 2, 3).

Line plots of percentage MR signal change along lateral sulcus. To visualize the fMRI responses during passive, active or observed touch along the upper bank of the lateral sulcus, we calculated percentage MR signal change in the voxels in steps of $1 \mathrm{~mm}$, from $y=+1 \mathrm{~mm}$ at the posterior end to $y=+22 \mathrm{~mm}$ at the anterior end of the lateral sulcus ( $y=0 \mathrm{~mm}$ corresponds to the position of the stereotactically positioned ear bars). These line plots were drawn for all four hemispheres. Percentage MR signal changes were plotted for following contrasts: hand stimulation versus ipsilateral face stimulation from the tactile localizer experiment, grasp execution versus reach execution from the motor localizer experiment, and grasp object observation versus fixation baseline from visual Experiment 2. Shaded regions flanking the line plots indicate variability across runs.

Multivariate analysis. In addition to the univariate analyses, we also performed a multivariate analysis on the functionally defined tactile SI and SII ROIs to investigate whether the voxel patterns of these functional 

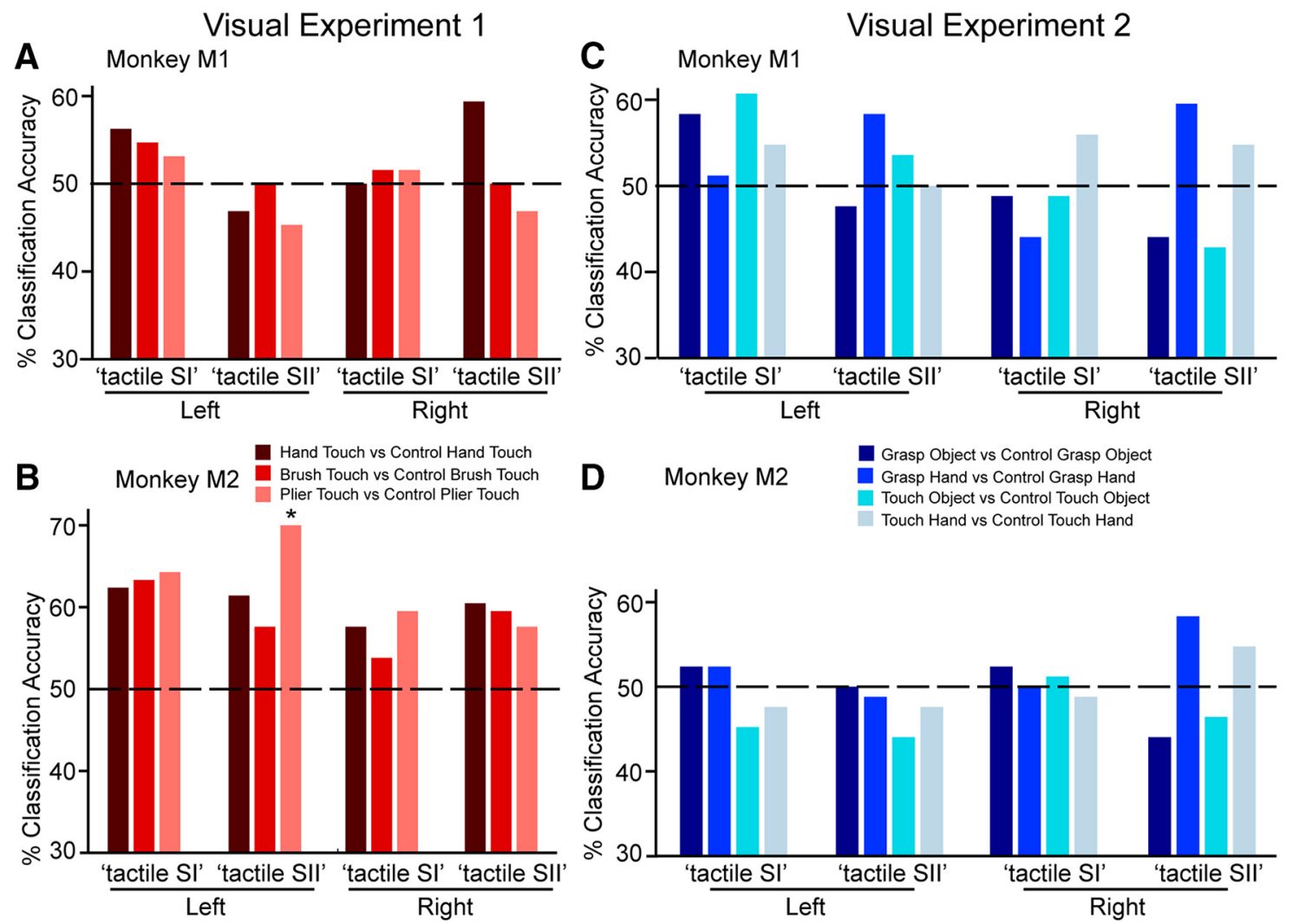

Figure 5. Multivariate fMRI decoding of observed touch in tactile SI and SII regions-of-interest. $A, B$, Classifier accuracies for binary decoding of observing a conspecific's hand being touched versus no-touch controls in tactile SI and SII of Monkey M1 ( $\boldsymbol{A}$ ) and Monkey M2 (B). Stippled lines indicate chance accuracy levels. Asterisk indicates significant decoding ( $p<0.05$, corr; see Materials and Methods). C, D, Classifier accuracies for binary decoding of observing a human hand grasping or touching an object or a hand (vs no-touch controls) in Monkey M1 (C) and Monkey M2 (D). Stippled lines indicate chance accuracy levels. All tested binary classifications yielded decoding accuracies not significantly different from chance level in tactile SI and SII, in both hemispheres of both monkeys.

ROIs allow discrimination between observed touch versus no touch. The $\beta$ estimates were extracted from left and right tactile SI and SII and used as inputs to a linear support vector machine classifier, as implemented in the decoding toolbox (Hebart et al., 2014) with a cost parameter $(c=1)$. We performed three pairwise binary decodings for visual Experiment 1 and four pairwise binary decodings for visual Experiment 2, in each case contrasting a touch condition with its respective no-touch control condition. A leave-one-run-out cross-validation was used to ensure generalizability of the results. At each iteration during the classification procedure, $\beta$ estimates of one run were used as test data while the classifier was trained on the rest of the $\beta$ estimates of the remaining runs (total runs: 32 runs for Monkey M1, 30 runs for Monkey M2 in visual Experiment 1; 42 runs for each monkey in visual Experiment 2). This was repeated for all runs such that each run served once as a test run and once as a training run. The decoding performance for all iterations was then averaged to obtain the decoding performance of each ROI. To determine the statistical significance of the decoding results, a permutation analysis was performed. For each ROI, the labels (or conditions of the visual experiments) were randomly shuffled to create 1000 unique labels. Classification on the permuted data followed the same cross-validation scheme as above. Based on the decoding performance values obtained for both the original classification and the permutations, $p$ values were estimated for each ROI. The $p$ values computed for all ROIs were corrected for multiple comparisons (number of ROIs) using false discovery rate (FDR). ROIs that gave $p$ values $<0.05$ after FDR correction were considered significant.

\section{Results}

We first localized the somatosensory hand representations in SI and SII in the individual monkeys using a passive tactile localizer experiment. While monkeys were lightly sedated in the scanner, we applied light cutaneous stimulation (using a brush) to either the palm or side of the face (Fig. 1A; see Materials and Methods). Tactile stimulation of the hand (compared with the ipsilateral face stimulation) yielded significant ( $p<0.05$, corr) contralateral brain responses in SI (in particular BA $3 \mathrm{~b}$ and 1 ) and SII somatosensory cortices (Fig. $2 A, B$; group data, $n=2$ ). Lowering the threshold ( $p<0.001$, uncorr) suggests similar functional responses also in/near area PFG on the inferior parietal lobule (Fig. 2A, B, insets). Additionally, we also examined fMRI responses while subjects actively grasped objects with the right hand (Fig. 2C,D), because previous research has shown that many somatosensory neurons are particularly responsive during active motor tasks (involving tactile and/or proprioceptive inputs), as opposed to passive stimulation (Fitzgerald et al., 2004; Ishida et al., 2013; Hihara et al., 2015). The grasping task was performed in the dark without visual feedback; hence, monkeys relied strongly on haptic feedback to complete this task. Consistent with previous monkey fMRI experiments using similar tasks (Nelissen and Vanduffel, 2011; Nelissen et al., 2018), grasping in the dark (compared with reaching-only) with the right hand yielded significant contralateral $\mathrm{fMRI}$ responses $(p<0.05$, corr $)$ throughout parietal areas AIP and PFG, primary motor area F1 (or M1), premotor F5, and ventrolateral prefrontal cortex (Fig. $2 C)$. In addition, significant responses were found in somatosensory cortices (Fig. 2C) including all portions of SI (BA 3a, 3b, 1, and 2), and more posteriorly, area 5. More ventrally, a large portion of the upper bank of the lateral sulcus also yielded significant 

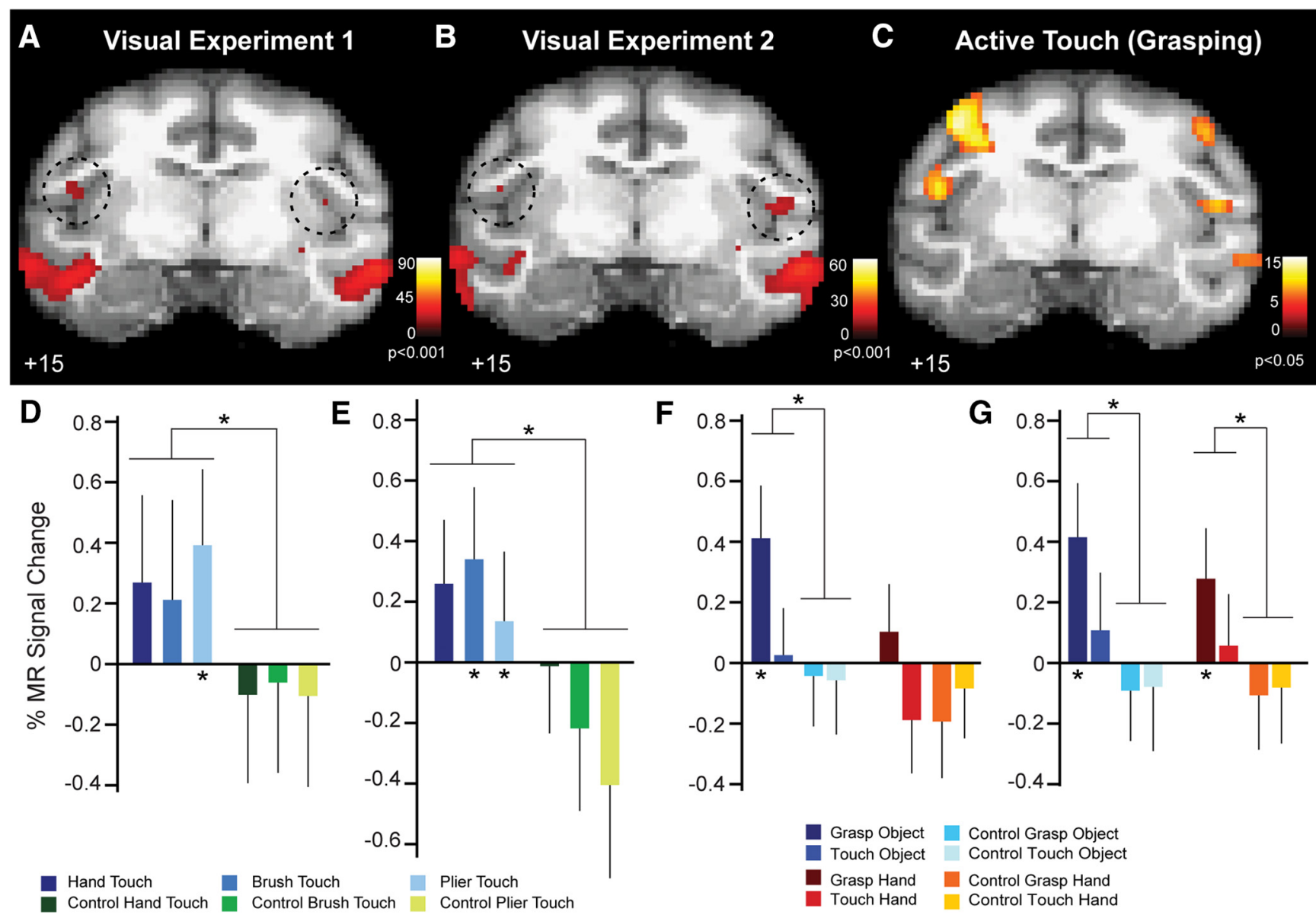

Figure 6. Functional MRI responses to observed and active touch in upper bank of lateral sulcus of Monkey M1. $A, B$, Coronal section showing fMRI responses during observation of touch (vs no-touch controls) in visual Experiment $1(\boldsymbol{A})$ and visual Experiment $2(\boldsymbol{B})$ in upper bank of the lateral sulcus (stippled black outlines) in Monkey M1. C, Coronal section showing fMRI responses during active touch (grasping in the dark) in the same location in the upper bank of lateral sulcus in Monkey M1. D, E, Percentage MR signal changes (compared with fixation-only baseline) in Monkey M1 for observing a conspecific's hand being touched and no-touch control videos in left $(\boldsymbol{D})$ and right $(\boldsymbol{E})$ local maxima of visual activations of the upper bank of the lateral sulcus shown in $\boldsymbol{A}$. Asterisks on top indicate a significant main effect for touch versus no touch (see Materials and Methods). Asterisks below horizontal axis indicate a significant response for each touch condition versus its respective no-touch control (Table 1).F, G, Percentage MR signal changes (compared with fixation-only baseline) in Monkey M1 for observing a human hand grasping or touching either an object or another hand and no-touch control videos in left $(\boldsymbol{F})$ and right $(\boldsymbol{G})$ local maxima of visual activations of the upper bank of the lateral sulcus shown in $\boldsymbol{B}$. Asterisks on top indicate significant main effects for either touch versus no touch directed toward objects or directed toward hands. Asterisks below horizontal axis indicate significant response for each touch condition versus its respective no-touch control (Tables 2,3).

fMRI responses, an activation largely overlapping with tactile SII as defined in the tactile localizer experiment (white outline), and extending both posteriorly into area $7 \mathrm{~b}$ (including area PFG), as well as anteriorly with respect to our tactile SII. In addition, the posterior portion of the insula also yielded robust contralateral grasping-related fMRI responses (Fig. 2C). Ipsilateral responses during grasp execution (compared with reach-only) were more restricted, including similar parietal, premotor, motor, prefrontal, somatosensory, and insular regions (Fig. 2D).

In Experiment 1, we determined whether observing a conspecific's hand being touched (Fig. 1C) would elicit fMRI responses in the somatosensory cortices of the monkey. Whole-brain analysis comparing all touch conditions (Fig. $1 E, G, I$ ) versus controls (Fig. $1 F, H, J$ ) yielded significant ( $p<0.05$, corr) brain responses in early visual and extrastriate cortices, extending toward the anterior tip of the temporal lobe, as well as in parietal, frontal, and prefrontal regions (Fig. $3 A, B$ ). In the lateral sulcus, bilateral responses were found in a small sector of the upper bank, anterior to our tactile SII (white outline), which we will refer to as visual SII. In addition, significant fMRI responses related to observing touch were found in the posterior portion of the insula (Fig. $3 A, B$ ).
Lowering the statistical threshold $(p<0.001$, uncorr) confirmed the absence of touch-observation related fMRI responses in tactile SI or SII (Fig. $3 A, B$, insets), and instead suggest these responses to be located both anterior (visual SII) and posterior (area PFG) to our tactile SII. Comparing all actions depicting touch from Experiment 2 versus their controls yielded comparable results as found in Experiment 1, showing significant $(p<$ 0.05 , corr) fMRI activations early visual and extrastriate visual regions, in addition to parietal, premotor, frontal, and prefrontal regions (Fig. 3C,D). As for Experiment 1, tactile SI and tactile SII (white outlines) did not yield touch-related fMRI responses. In the lateral sulcus, visual responses for touch observation (vs controls) were instead present anterior to tactile SII, in the same location (visual SII) where visual responses were also found in Experiment 1 (Fig. 3C,D, insets; $p<0.001$, uncorr). Finally, at the group level, visual responses contrasting all touch conditions versus controls in Experiment 2 yielded differential responses only in the right posterior insula (Fig. $3 C, D$ ), as opposed to the bilateral posterior insular responses for touch observation found in Experiment 1. Single subject analysis (see Figs. 8 and 9) showed that this was largely due to unilateral visual responses in the insula of Monkey M1. 


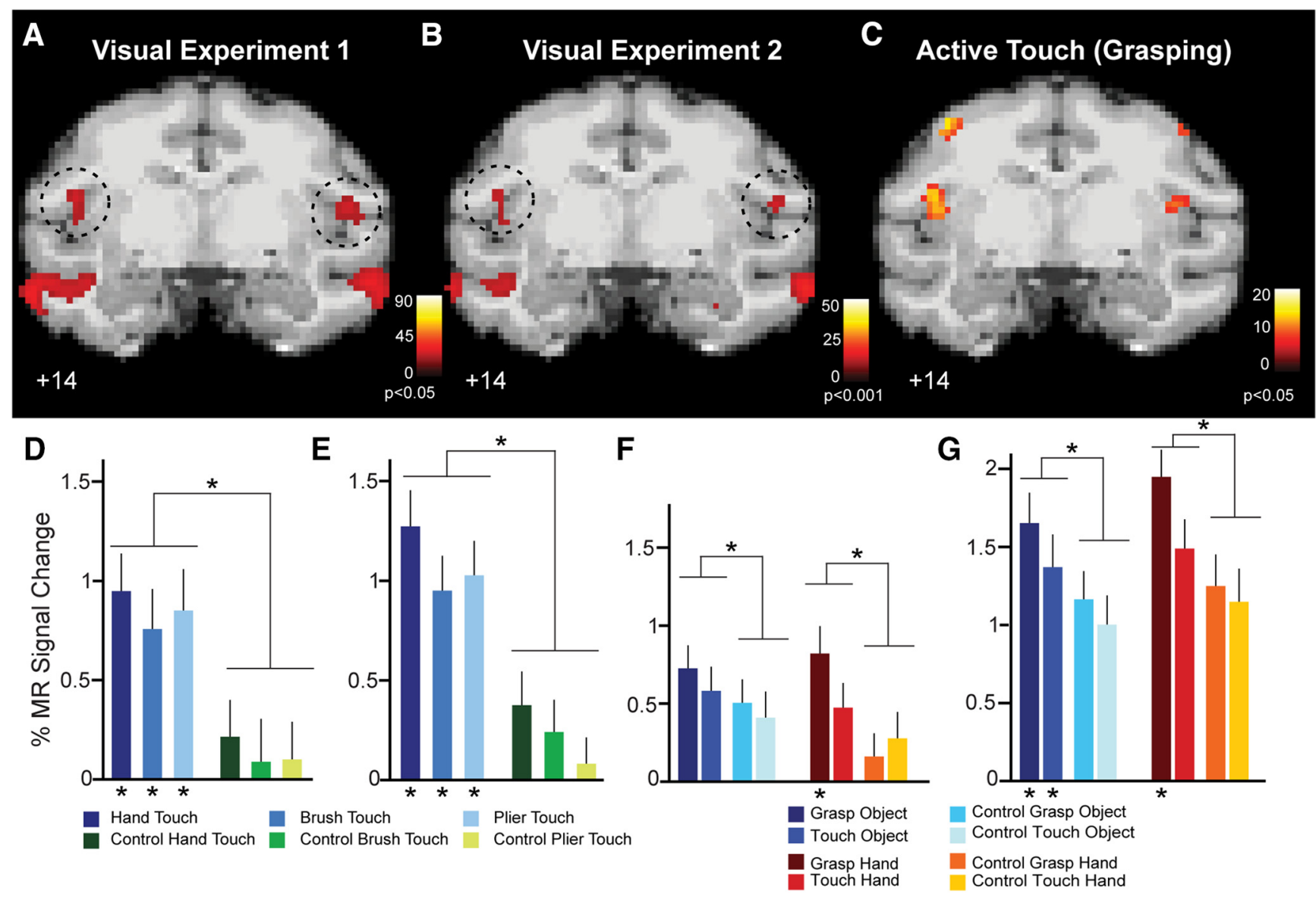

Figure 7. Functional MRI responses to observed and active touch in upper bank of lateral sulcus of Monkey M2. $A, B$, Coronal section showing fMRI responses during observation of touch (vs no-touch controls) in visual Experiment $1(\boldsymbol{A})$ and visual Experiment 2 (B) in upper bank of the lateral sulcus (stippled black outlines) in Monkey M2. C, Coronal section showing fMRI responses during active touch (grasping in the dark) in the same location in the upper bank of the lateral sulcus of Monkey M2. D, E, Percentage MR signal changes (compared with fixation-only baseline) in Monkey M2 for observing a conspecific's hand being touched and no-touch control videos in left (D) and right $(\boldsymbol{E})$ local maxima of visual activations of the upper bank of the lateral sulcus shown in $\boldsymbol{A}$. Asterisks on top indicate a significant main effect for touch versus no touch. Asterisks below horizontal axis indicate a significant response for each touch condition versus its respective no-touch control (Table 1). $F, G$, Percentage MR signal changes (compared with fixation-only baseline) in Monkey $M 2$ for observing a human hand grasping or touching either an object or another hand and no-touch control videos, in left $(\boldsymbol{F})$ and right $(\boldsymbol{G})$ local maxima of visual activations of the upper bank of the lateral sulcus shown in $\boldsymbol{B}$. Asterisks on top indicate significant main effects for either touch versus no touch directed toward objects or directed toward hands. Asterisks below horizontal axis indicate significant response for each touch condition versus its respective no-touch control (Tables 2,3).

To examine the possible presence of vicarious touch responses in somatosensory cortices in greater detail, we also performed a ROI analysis of our functionally defined tactile SI and tactile SII hand regions. As shown in Figure 4, ROI analysis suggests that both left and right tactile SI and tactile SII regions did not yield significant visual responses (compared with fixation baseline). This was the case for both individual subjects, in Experiment 1 (Fig. $4 A, B$ ), as well as in Experiment 2 (Fig. 4C,D).

In addition to this univariate ROI analysis, we performed a multivoxel analysis in the tactile SI and SII ROIs to determine whether observation of touch (compared with no touch) might be represented in the finer-grained spatial patterns within tactile SI or SI. Therefore, binary classifiers were constructed, testing pairwise decoding of all touch conditions versus their respective controls for visual Experiments 1 and 2. Overall, classifiers failed to find significant distinctions between the touch observation versus no touch observation voxels patterns from bilateral tactile SI and SII (Fig. 5). With the exception of "pliers touch" versus "control pliers touch" from Experiment 1 in left "tactile SII" of Monkey M2 ( $p=0.012$, corrected), all other binary comparisons (touch observation conditions vs their respective notouch controls) yielded decoding accuracies not significantly different from chance level (Experiment 1: Fig. 5A,B; Experiment 2: $C, D)$.

We next examined in more detail the visual responses observed in the lateral sulcus (visual SII) and in the posterior insula (Fig. 3). We plotted MR signal changes from the local maxima of these fMRI activations, observed in Experiment 1 and Experiment 2, for each subject separately. The location of these visual activations in the upper bank of the lateral sulcus [Monkey M1: Experiment 1, Fig. 6A; Experiment $2(B)$; Monkey M2: Experiment 1, Fig. 7A; Experiment 2, $(B)$ ] overlapped with a region in the lateral sulcus that also yielded significant MR signal changes during grasping execution (Monkey M1: Fig. 6C; Monkey M2: Fig. 7C). Functional MR responses to the observation of a conspecific's hand being touched with different effectors, compared with no-touch controls (Experiment 1), yielded significant responses in both monkeys [main effect touch vs no touch, Monkey M1: Fig. $6 D$, left hemisphere: $F_{(1,31)}=8.926 ; p=0.005$; $(E)$ right hemisphere: $F_{(1,31)}=12.21 ; p=0.0015$; Monkey M2: Fig. $7 D$, left hemisphere: $F_{(1,29)}=115.4 ; p=1.3 \times 10^{-11} ;(E)$ right hemisphere: $F_{(1,29)}=226.5 ; p=3 \times 10^{-15}$ ]. Post hoc tests directly comparing responses to observation of touch with different effectors, did not reveal any significant differences between the three 


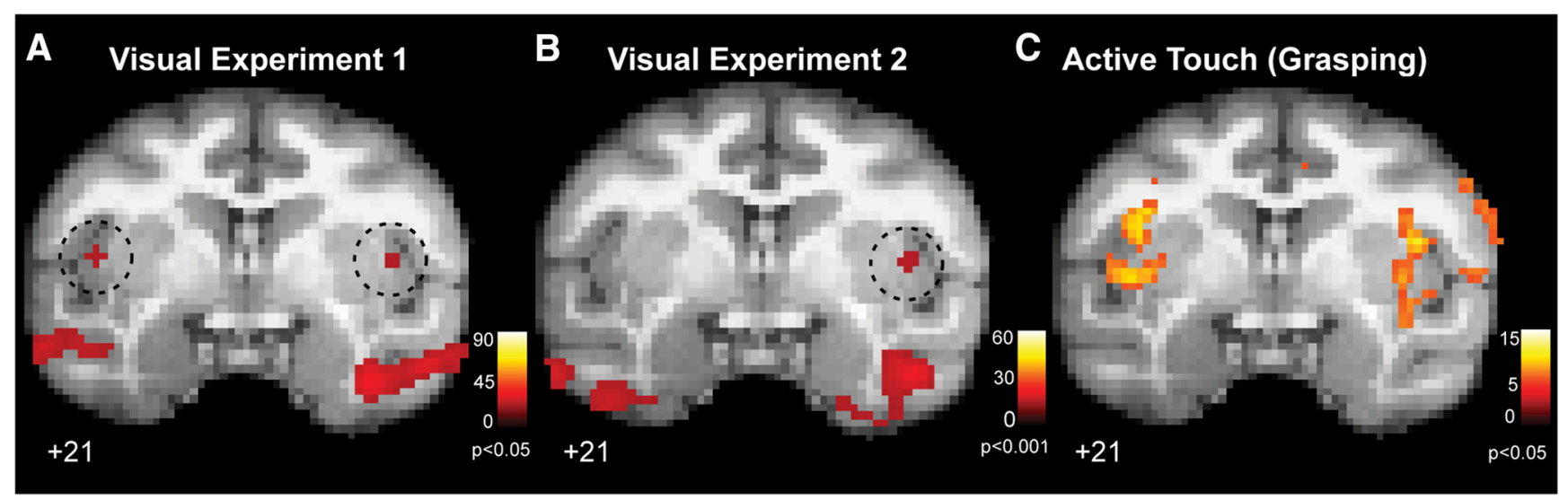

D

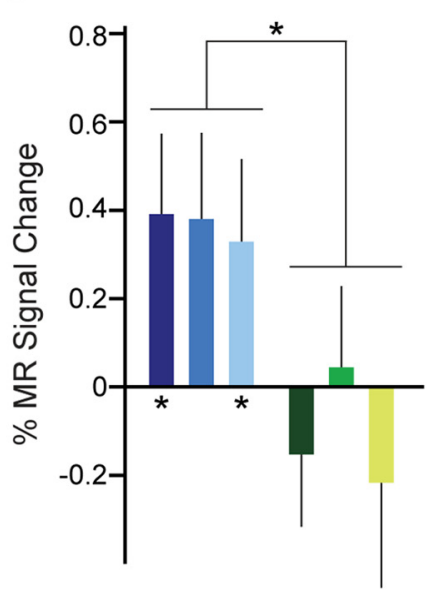

E

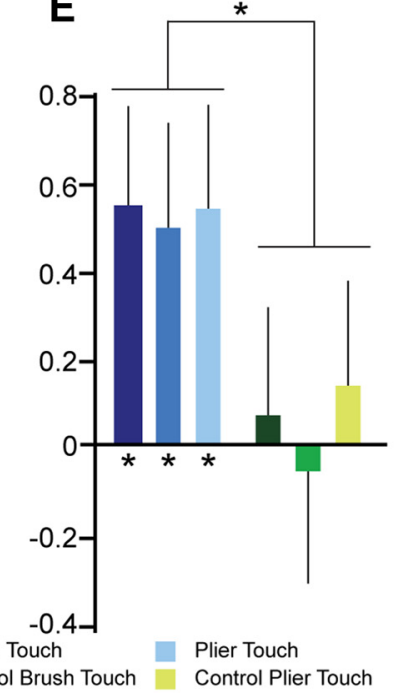

F

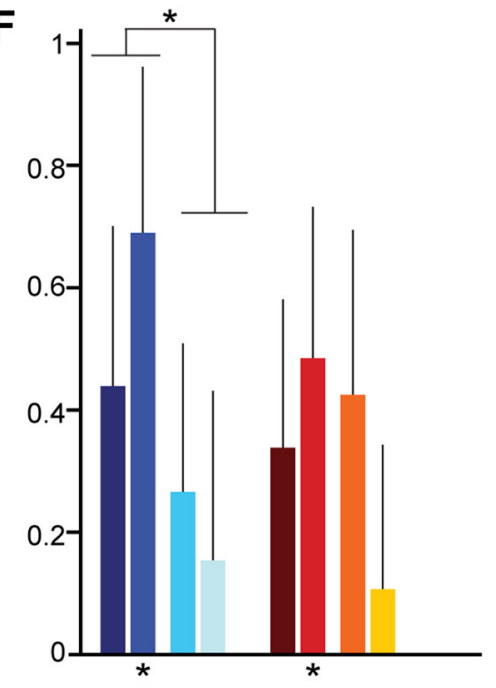

Grasp Object

Touch Object

Control Grasp Object

Control Touch Object

Grasp Hand

Touch Hand

Control Grasp Hand

Control Touch Hand

Figure 8. Functional MRI responses to observed and active touch in posterior insula of Monkey M1. $\boldsymbol{A}, \boldsymbol{B}$, Coronal section showing fMRI responses during observation of touch (vs no-touch controls) in visual Experiment $1(\boldsymbol{A})$ and visual Experiment $2(\boldsymbol{B})$ in posterior insula (stippled outlines) in Monkey M1. $\boldsymbol{C}$, Coronal section showing fMRI responses during active touch (grasping in the dark) in in the same location in the posterior insula in Monkey M1. D, $\boldsymbol{E}$, Percentage MR signal changes (compared with fixation-only baseline) in Monkey M1 for observing a conspecific's hand being touched and no-touch control videos in left $(\boldsymbol{D})$ and right $(\boldsymbol{E})$ local maxima of posterior insula activations shown in $\boldsymbol{A}$. Asterisks on top indicate a significant main effect for touch versus no touch. Asterisks below horizontal axis indicate a significant response for each touch condition versus its respective no-touch control (Table 1). $\boldsymbol{F}$, Percentage MR signal changes (compared with fixation-only baseline) in Monkey M1 for observing a human hand grasping or touching an object or another human hand, and no-touch control videos in right local maximum of posterior insula activation shown in $\boldsymbol{B}$. Asterisks on top indicate significant main effects for either touch versus no touch directed toward objects or directed toward hands. Asterisks below horizontal axis indicate significant response for each touch condition versus its respective no-touch control (Tables 2,3).

effectors in left and right visual SII of Monkey M1 and left visual SII of Monkey M2. In right visual SII of Monkey M2 (Fig. 7E), observation of a conspecific's hand being touched by a biological effector (human hand) yielded significantly stronger responses compared with observation of touch by a brush $(p=0.02)$ or pliers $(p=0.005)$.

For Experiment 2, we investigated the responses of visual SII for observing actions directed toward an object versus their controls, and for observing actions directed toward a human hand versus their controls. In the four hemispheres, visual SII yielded a main effect for observing actions directed toward an object (grasping an object + touching an object) versus no-touch controls [Monkey M1: Fig. $6 F$, left hemisphere: $F_{(1,41)}=6.946 ; p=$ $0.012 ;(G)$ right hemisphere: $F_{(1,41)}=12.25 ; p=0.0011$; Monkey M2: Fig. $7 F$, left hemisphere: $F_{(1,41)}=7.97 ; p=0.0073$; $(G)$ right hemisphere: $\left.F_{(1,41)}=13.66 ; p=0.0006\right]$. In addition, three of the four hemispheres showed a similar main effect for observing human hands being grasped or touched, versus no-touch controls (Monkey M1: Fig. $6 F$, left hemisphere: $F_{(1,41)}=0.82 ; p=0.37$; $(G)$ right hemisphere: $F_{(1,41)}=8.043 ; p=0.0072$; Monkey M2:
Fig. $7 F$, left hemisphere: $F_{(1,41)}=28.14 ; p=4 \times 10^{-6} ;(G)$ right hemisphere: $\left.F_{(1,41)}=17.85 ; p=0.0001\right)$. Post hoc tests also showed that responses for observing a hand grasping versus touching an object were only significantly different in left visual SII of Monkey M1 ( $p=0.034)$. The same comparison for observing a hand grasping another hand or mere touching it, only yielded significant different responses in right visual SII of Monkey M2 ( $p=0.0189)$.

The posterior insula also yielded significant visual responses in both visual experiments (Monkey M1: Fig. $8 A, B$; black stippled outline; Monkey M2: Fig. 9 A, B; black stippled outline). The motor localizer experiment showed that at the corresponding location, active touch (when grasping an object in the dark) also evoked significant signal increases (Monkey M1: Fig. 8C; Monkey M2: Fig. 9C). Observing a conspecific's hand being touched compared with no-touch controls yielded a significant mean effect of touch in both left and right posterior insula of Monkey M1 [Fig. $8 D$, left hemisphere: $F_{(1,31)}=32.89 ; p=0.00003$; $(E)$ right hemisphere: $F_{(1,31)}=27.28 ; p=0.00001$ ] and Monkey M2 [Fig. 9D, left hemisphere: $F_{(1,29)}=71.01 ; p=2.89 \times 10^{-9} ;(E)$ right hemi- 

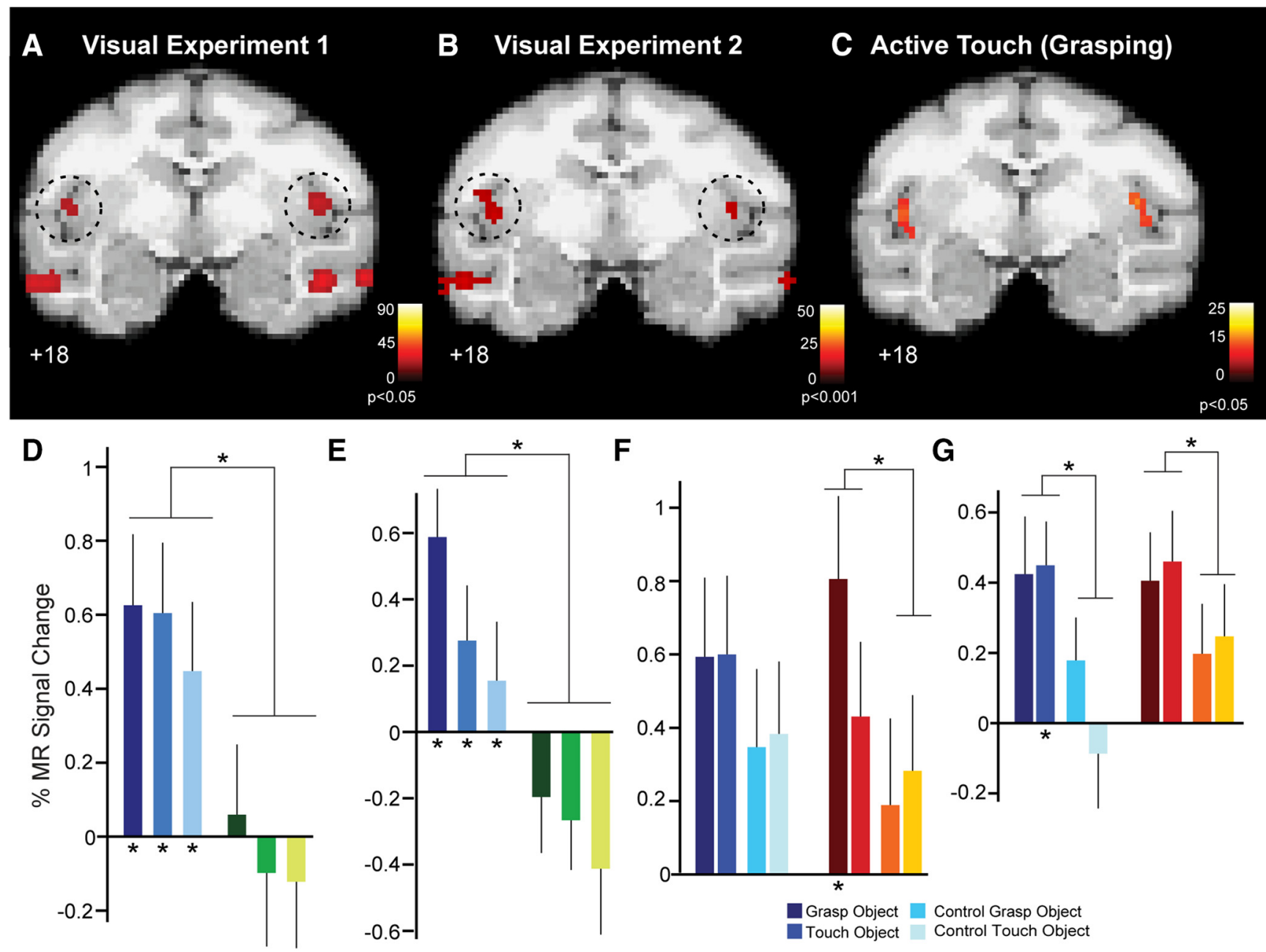

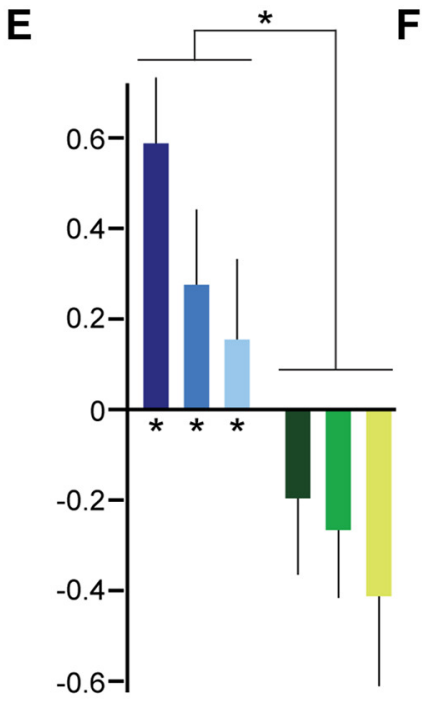

F

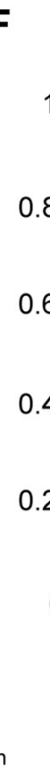

G

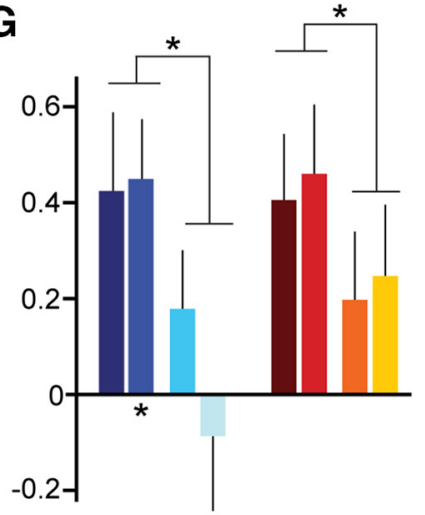

Figure 9. Functional MRI responses to observed and active touch in posterior insula of Monkey M2. $\boldsymbol{A}, \boldsymbol{B}$, Coronal section showing fMRI responses during observation of touch (vs no-touch controls) in visual Experiment $1(\boldsymbol{A})$ and visual Experiment $2(\boldsymbol{B})$ in posterior insula (stippled outlines) in Monkey M2. $\boldsymbol{C}$, Coronal section showing fMRI responses during active touch (grasping in the dark) in the same location in the posterior insula of Monkey M2. D, E, Percentage MR signal changes (compared with fixation-only baseline) in Monkey M2 for observing a conspecific's hand being touched and no-touch control videos in left $(\boldsymbol{D})$ and right $(\boldsymbol{E})$ local maxima of posterior insula activations shown in $\boldsymbol{A}$. Asterisks on top indicate a significant main effect for touch versus no touch. Asterisks below horizontal axis indicate a significant response for each touch condition versus its respective no-touch control (Table 1). $\boldsymbol{F}, \mathbf{G}$, Percentage MR signal changes (compared with fixation-only baseline) in Monkey M2 for observing a human hand grasping or touching an object or another human hand, and no-touch control videos in left (F) and right ( $\boldsymbol{G}$ ) local maxima of posterior insula activation shown in $\boldsymbol{B}$. Asterisks on top indicate significant main effects for either touch versus no touch directed toward objects or directed toward hands. Asterisks below horizontal axis indicate significant response for each touch condition versus its respective no-touch control (Tables 2, 3).

sphere: $\left.F_{(1,29)}=77.09 ; p=1.2 \times 10^{-9}\right]$. Post hoc tests did not reveal significant differences between observing touch with different effectors, except for hand- versus plier-touch in right insula of Monkey M2 (Fig. 9E; $p=0.001$ ).

In Experiment 2, the posterior insula yielded a main effect for observing actions directed toward an object versus controls (grasping an object + touching an object vs no-touch controls) in right hemispheres of Monkey M1 (Fig. 8F; $F_{(1,41)}=5.07 ; p=0.03$ ) and Monkey M2 (Fig. 9F; $F_{(1,41)}=19.97 ; p=0.000061$ ). A similar main effect for observing a human hand being grasped or touched (vs no-touch controls) was also observed in both hemispheres of Monkey M2 (Fig. 9F, left hemisphere: $F_{(1,41)}=22.8$; $p=0.000023 ;(G)$ right hemisphere: $\left.F_{(1,41)}=6.04 ; p=0.018\right)$. Post hoc test comparing directly responses to observing grasping versus observing touching did not reveal any significant differences, nor for actions directed to objects, nor for actions directed to hands.
Posterior to tactile SII, the rostral portion of the inferior parietal lobule also yielded MR responses during passive tactile stimulation (Fig. $2 A, B$, insets) and during active grasp execution (Fig. $2 C, D$, insets). This region, presumably corresponding to (part of) area PFG, also responded to touch observation (Figs. 10, 11). A main effect of observing touch (vs controls) was observed particularly in the right hemisphere of both Monkey M1 (Fig. 10C; right hemisphere: $F_{(1,31)}=31.78, p=0.000003$ ) and Monkey M2 (Fig. $11 C$; right hemisphere: $F_{(1,29)}=79.83, p=7.96 \times 10^{-10}$ ) for Experiment 1. Post hoc tests did not reveal significant differences between observing touch with different types of effectors.

Observing a human hand grasping or touching objects (Experiment 2) also yielded significant main effects of touch (vs controls) in the right hemispheres of Monkey M1 (Fig. 10D; right hemisphere: $F_{(1,41)}=8.402, p=0.006$ ) and Monkey M2 (Fig. $11 E$; right hemisphere: $F_{(1,41)}=5.72, p=0.022$ ). In addition, area PFG yielded a main effect for observing touch for observing hu- 
man hands being grasped or touched in the right hemisphere of Monkey M1 (Fig. $10 D$; right hemisphere: $F_{(1,41)}=5.914$, $p=0.019)$ and both hemispheres of Monkey M2 [Fig. 11D; left hemisphere: $F_{(1,41)}=$ $17.92 ; p=0.0001 ;(E)$ right hemisphere: $F_{(1,41)}=53.3, p=6.2 \times 10^{-9}$ ]. Post hoc test comparing grasping versus touching for actions directed to objects only revealed a significant difference in right PFG of Monkey M2 ( $p=0.002)$. For actions directed to hands, grasping versus touching yielded significantly different responses in both hemispheres of Monkey M2 (left: $p=0.0196$; right: $p=9.8 \times$ $\left.10^{-6}\right)$.

Finally, to directly compare the extent and location of the fMRI responses found in our localizer and visual experiments in SII and surrounding cortex, we investigated the fMRI responses along the posterior-anterior extent of the upper bank of the lateral sulcus. We plotted percentage MR signal change elicited during passive tactile stimulation (contralateral hand stimulation vs face stimulation), active touch (grasp execution vs reach execution with the right hand) and observed touch (observing a human hand grasping an object vs no-touch control only baseline, Experiment 2) along a path in the upper bank of the lateral sulcus, starting posterior $(y=+2 \mathrm{~mm})$ toward the anterior end $(y=+22 \mathrm{~mm})$, in both hemispheres of both subjects (Monkey M1: Fig. 12A,B: Monkey M2: C,D). Tactile responses during passive cutaneous stimulation of the contralateral hand were particularly evident in a confined sector of the upper bank (blue line). Active touch during grasp execution (with the right hand) yielded an extended activation, overlapping with the portion of the lateral sulcus also activated during passive touch, but extending both more posteriorly and anteriorly (green line). It should be noted that grasping-related responses in left and right hemisphere might not be straightforward comparable because they reflect contralateral versus ipsilateral responses in respective left and right hemispheres. Visual responses to grasp observation were most obvious in a sector of the upper bank (red line), anterior to the focus where passive tactile stimulation evoked its strongest responses. This visually responsive portion of the lateral sulcus also yielded strong responses during grasping execution (Fig. 12).

\section{Discussion}

Possible correspondence of our functionally defined SII regions to previous literature

The exact functional organization of the somatosensory cortices in the upper bank of the lateral sulcus of the macaque monkey is not yet completely understood. Macaque SII was initially described as a single field (Friedman et al., 1980; Pons et al., 1988),
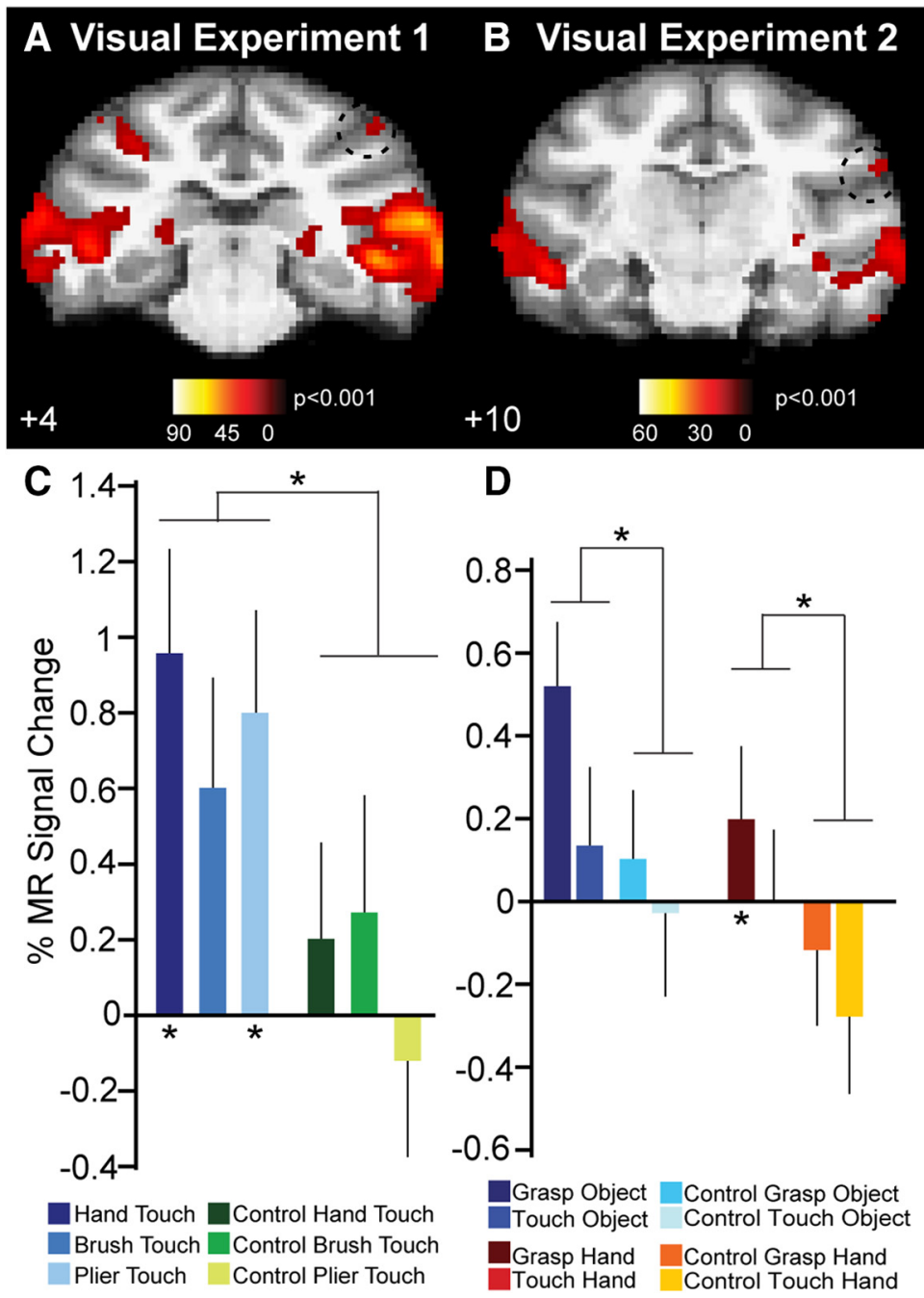

Figure 10. Functional MRI responses to observed touch area PFG of Monkey M1. $\boldsymbol{A}, \boldsymbol{B}$, Coronal section showing fMRI responses during observation of touch (vs no-touch controls) in visual Experiment $1(\boldsymbol{A})$ and visual Experiment $2(\boldsymbol{B})$ in area PFG (stippled (n) Monkey M1. C, Percentage MR signal changes (compared with fixation-only baseline) in Monkey M1 for observing a p indicate a significant main effect for touch versus no touch. Asterisks below horizontal axis indicate a significant response for only baseline) in Monkey M1 for observing a human hand grasping or touching an object or another human hand, and no-touch control videos in right local maximum of area PFG activation shown in $\boldsymbol{B}$. Asterisks on top indicate significant main effects for either touch versus no touch directed toward objects or directed toward hands. Asterisks below horizontal axis indicate significant response for each touch condition versus its respective no-touch control (Tables 2,3).

but others later suggested that SII consisted of two (Burton et al., 1995; Krubitzer et al., 1995) or even three subregions (Fitzgerald et al., 2004). Whereas Burton and coauthors (1995) suggested two neighboring body map representations in SII (posterior SII and anterior SII), Krubitzer et al. (1995) referred to two posterior and anterior mirror body representations termed, respectively, area SII and area PV, in line with similar terminology used in other species. Fitzgerald et al. (2004) on the other hand proposed that macaque SII comprised a posterior, central, and anterior subregion. These authors suggested that their posterior and central SII subregions might correspond to SII as defined by Krubitzer et al. (1995), whereas their anterior SII subregion might correspond to area PV of Krubitzer et al. (1995).

It is difficult, however, to make conclusive statements about the correspondence of our functionally defined tactile SII with these previous suggested partitioning schemes of SII. Fitzgerald et 

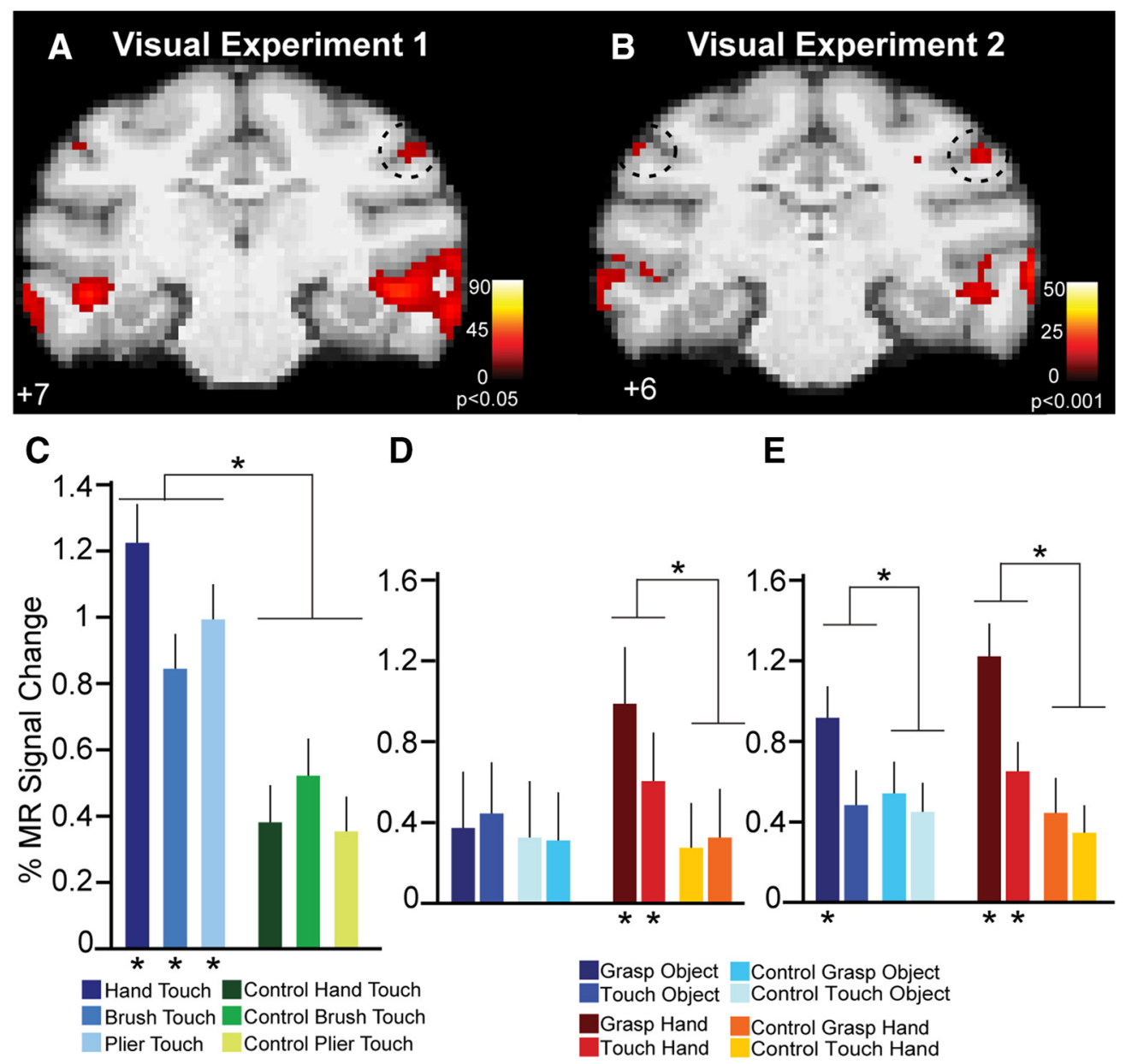

Figure 11. Functional MRI responses to observed and active touch in area PFG of Monkey M2.A, $B$, Coronal section showing fMRI responses during observation of touch (vs no-touch controls) in visual Experiment $1(\boldsymbol{A})$ and visual Experiment $2(\boldsymbol{B})$ in area PFG (stippled outlines) in Monkey M2. C, Percentage MR signal changes (compared with fixation-only baseline) in Monkey M2 for observing a conspecific's hand being touched and no-touch control videos in right local maximum of area PFG activation shown in $A$. Asterisks on top indicate a significant main effect for touch versus no touch. Asterisks below horizontal axis indicate a significant response for each touch condition versus its respective no-touch control (Table 1). $\boldsymbol{D}, \boldsymbol{E}$, Percentage MR signal changes (compared with fixation-only baseline) in Monkey M2 for observing a human hand grasping or touching an object or another human hand, and no-touch control videos in left $(\boldsymbol{D})$ and right $(\boldsymbol{E})$ local maxima of area PFG activations shown in $\boldsymbol{B}$. Asterisks on top indicate significant main effects for either touch versus no touch directed toward objects or directed toward hands. Asterisks below horizontal axis indicate significant response for each touch condition versus its respective no-touch control (Tables 2,3).

al. (2004) suggested that SII consisted of three zones, a central zone responding well to passive cutaneous stimulation (i.e., light brushing), in addition to posterior and anterior zones responding particularly well to proprioceptive stimulation or when monkeys performed active movements, especially when grasping objects. An arrangement similar to that proposed by Fitzgerald et al. (2004), can also be seen in our data. In all four hemispheres examined in our study, we found a region (tactile SII) in the upper bank of the lateral sulcus that responded during passive cutaneous stimulation of the hand (Fig. $2 A, B$ ). This region was flanked anteriorly and posteriorly by additional fields that instead of responding during passive cutaneous stimulation, displayed strong increases in fMRI signal during active cutaneous and proprioceptive stimulation, when monkeys grasped objects in the dark using haptic feedback (Fig. 2C,D). Possibly our visual II would be located in/near the anterior SII zone of Fitzgerald et al. (2004), which, according to those authors, might correspond to area PV as defined by Krubitzer et al. (1995). Another possibility is that our tactile SII corresponds to both the neighboring hand fields of SII and PV, as defined by Krubitzer et al. (1995), or (portion of) the three subfields of SII according to Fitzgerald et al. (2004). If this is the case, then our visual SII, that also yielded motor responses, might be located in/near area PR. This interpretation would fit with the terminology used in a human fMRI/ MEG study, describing responses in presumed human SII/PV during tactile stimulation and more anterior responses, in a region termed $\mathrm{PR}$, during tactile and proprioceptive stimulation involving closing a hand around an object (Hinkley et al., 2007). Future studies investigating the connections of our functionally defined regions, in addition to a detailed mapping of the functional properties of the underlying neurons, will be needed to further characterize the correspondence of our functionally defined SII regions/fields with regard to previous work (Kaas, 1993; Krubitzer et al., 1995; Disbrow et al., 2003; Fitzgerald et al., 2004).

\section{Visual responses in primary and secondary somatosensory cortices}

Traditionally, SI and SII cortices have been considered unimodal regions devoted to processing tactile and proprioceptive information for object recognition and motor control. In monkeys, it has been shown using the 2-deoxyglucose technique that observing and executing grasping movements modulates activity in somatosensory cortices (Raos et al., 2004; Evangeliou et al., 2009; Raos and Savaki, 2016). A recent study in awake monkeys specif- 

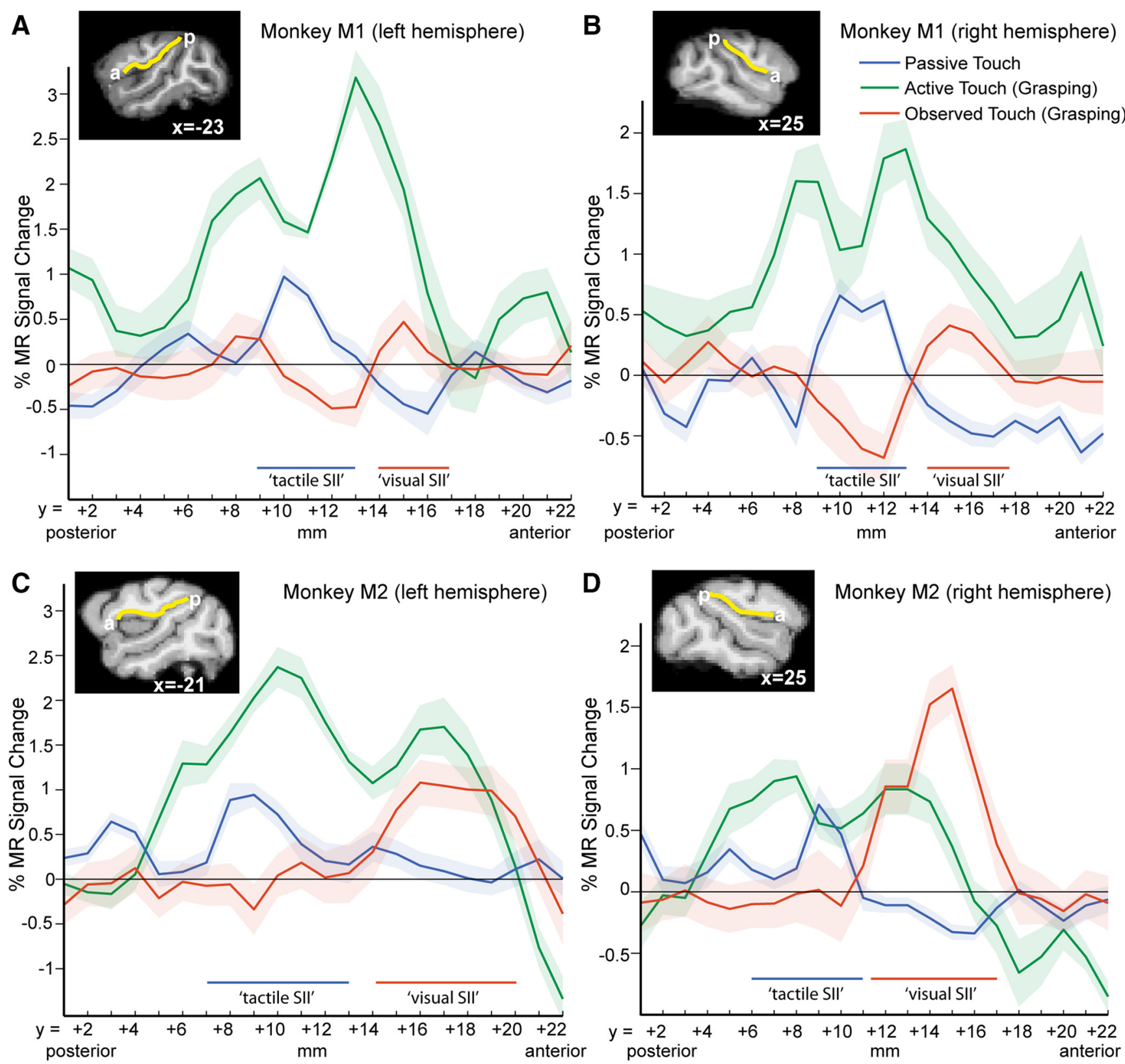

Figure 12. Functional MRI responses to passive, active and observed touch along the upper bank of the lateral sulcus. $\boldsymbol{A}-\boldsymbol{D}$, Line plots showing MR percentage signal change related to passive touch (cutaneous stimulation of contralateral hand vs face; blue line), active touch (grasp vs reach-only execution with the right hand; green line), and observed touch (Experiment 2, object grasp observation vs no-touch control; red line), along the length of the upper bank of the lateral sulcus in left and right hemispheres of Monkeys M1 (A, B) and M2 (C, $\boldsymbol{D})$. On the abscissa, the $y$-coordinates indicate the posterior-anterior extent of the path, starting near the posterior ( $\mathrm{p}$ ) side at the level of area PFG, toward the anterior (a) end of the lateral sulcus, as indicated in the sagittal insets. Shaded regions flanking the line plots indicate variability across runs.

ically examined visual responses in SII and surrounding cortex at the single-cell level (Hihara et al., 2015). These authors found that approximately one-fourth of the recorded neurons modulated their responses during visual stimulation. It is interesting that the authors reported that neurons responding during observation of human actions were also more frequently active during active self-movements compared with passive somatosensory stimulation (Hihara et al., 2015). This observation seems to fit with our finding that the region anterior to tactile SII, yielding action/touch observation-related visual responses also yielded responses during active self-movements as opposed to passive cutaneous stimulation (Fig. 12).

Interestingly, visual stimuli seem to elicit responses in only a part of human SII (Bremmer et al., 2001; Keysers et al., 2004). The suggestion that human SII might be composed of a purely somatosensory and a second, multimodal part (Keysers et al., 2004) fits with our observation of passive tactile responses in tactile SII and visual/active tactile/proprioceptive responses in the more anterior cluster (visual SII). A better understanding of the connectivities of our functionally defined regions, in addition to a detailed mapping of the functional properties of the underlying neurons, will be needed to further characterize the correspondence of our functionally defined SII regions/fields with regard to previous investigations in monkeys (Burton et al., 1995; Krubitzer et al., 1995; Disbrow et al., 2003; Fitzgerald et al., 2004).

Our finding of minimal engagement, particularly by monkey SI, during touch observation might seem at odds with several 
human studies (Blakemore et al., 2005; Ebisch et al., 2008; Schaefer et al., 2009; Meyer et al., 2011; Kuehn et al., 2013). With respect to vicarious responses in human somatosensory cortices, it has been suggested that these might either reflect the mirroring or simulation of the somatosensory aspects of seeing someone performing a certain action, or on the other hand might be related to mirroring the somatosensory states of others (Keysers et al., 2010). Because our current study was not designed to specifically address this possible distinction, it remains an open question whether a similar distinction exists in monkey somatosensory cortices. Recent data from our group, using cross-modal multivoxel classification analysis of fMRI data in monkeys either performing or observing motor acts (Fiave et al., 2017, SFN abstract, 62.06/EE11), suggest that this first type of simulation (vicarious responses in somatosensory cortices when seeing someone performing a certain action), indeed seem to be present in somatosensory cortex.

It should be noted that particularly with regard to this second type of vicarious response (mirroring the somatosensory state of others), there is currently little consensus concerning the exact contributions of human SI, SII, and possible additional somatosensory regions, in mirroring touch. Although some authors point to involvement of (portions of) SI during touch observation (Blakemore et al., 2005; Bufalari et al., 2007; Schaefer et al., 2009; Pihko et al., 2010; Meyer et al., 2011; Rossetti et al., 2012; Kuehn et al., 2013), others suggest the specific involvement of SII (Keysers et al., 2004), or of both regions (Ebisch et al., 2008). More recently, others failed to find responses for merely observing touch in SI and SII (Chan and Baker, 2015; Ferri et al., 2015). Such inconsistent results regarding touch mirroring in human somatosensory cortices have been explained by discrepancies in stimuli: depicting active versus passive touch, intentional versus nonintentional touch, discriminative versus affective touch, mere touch versus skin displacements, etc.

Although differences in human and monkey somatosensory cortices during touch observation might be attributable to genuine species differences (but see Chan and Baker, 2015), an important factor to consider here is the amount of previous sensorimotor experience subjects may have had with the actions depicted in the videos. Sensorimotor experience has been shown to play a role in the emergence of shared responses in the mirror neuron system, both in humans (Catmur et al., 2007; Heyes, 2010; Press et al., 2012) and in monkeys (Ferrari et al., 2005). Zhou and Fuster (2000) showed that behavioral training can result in SI neurons that respond to visual stimuli associated with the touch of an object through associative learning of visual cues and haptic tasks. Also, Shokur et al. (2013) showed that SI neurons increase their firing rates when observing a virtual avatar arm being touched, following a period of association training during which virtual touches occurred synchronously with physical brushes of the monkeys' own arm. Therefore, it is possible that prior physical experience with the actions depicting touch shown in the videos (for instance observing one's own hand being touched as in Experiment1), might lead to stronger visual responses from SI and SII cortices when later on observing these actions. Future experiments should specifically test this proposal in monkeys by examining experience-induced changes in fMRI responses (Goda et al., 2016) in somatosensory cortex, after monkeys acquired simultaneous tactile and visual experience with a brush touching their hand, for instance.

\section{Vicarious touch responses in monkey PFG and insula?}

In addition to robust responses in tactile SI and SII, our tactile localizer experiment also yielded MR responses, during passive cutaneous stimulation of the contralateral hand, in area PFG on the inferior parietal convexity (Fig. 2A,B, insets). The same region, particularly in the contralateral hemisphere, was also activated during active grasping movements (Fig. 2C, inset), consistent with its suggested role in the fine control of object grasping and manipulation (Borra et al., 2017). These fMRI observations fit well with single-cell studies, suggesting that area PFG contains motor neurons and somatosensory neurons responding during active grasping movements (Fogassi et al., 2005; Rozzi et al., 2008) or cutaneous stimulation of the hand (Rozzi et al., 2008). Single-unit recordings have shown that area PFG also contains many neurons with visual properties (Rozzi et al., 2008). Of particular importance with respect to our study is that area PFG contains mirror neurons, responding not only during monkeys' own grasping movements, but also during observing the grasping actions of others (Fogassi et al., 2005; Rozzi et al., 2008; Bonini et al., 2010). In line with these single-cell and previous monkey fMRI (Nelissen et al., 2011) investigations, observation of a grasping motor act directed toward an object or hand (Experiment 2) indeed appears to elicit strong responses in area PFG (Monkey M1: Fig. 10D; Monkey M2: Fig. 11D,E). Although no direct comparison has been made at the single-cell level between visual responses during grasp observation versus mere touch observation, our data from Experiment 1 suggests that merely observing a conspecific hand being touched by different biological or nonbiological effectors, also yields robust fMRI responses in this region (Monkey M1: Fig. 10C; Monkey M2: Fig. 11C). Interestingly, a recent human fMRI study also found tactile, motor and visual responses related to touch observation in a region of the inferior parietal cortex, that may correspond to macaque PFG (Chan and Baker, 2015).

Our finding of fMRI modulations in the posterior-dorsal portion of the insula by active grasping (Fig. $2 C, D$ ) is in agreement with electrophysiological, connectivity and stimulation data showing that this portion of the insula contains a sensory-motor field, and is involved in somatosensory and motor processing (Robinson and Burton, 1980; Schneider et al., 1993; Jezzini et al., 2012; Ishida et al., 2013). Because we did not have cytoarchitectonic information from either subject, we refer to this portion of insular cortex as posterior insula. Interestingly, Ishida et al. (2013) showed this portion of the cortex contained neurons selectively activated during specific types of hand-manipulation tasks including the finger exploration and precision grasping common in the grooming behavior of monkeys. Preliminary data from the same authors suggest the presence of grooming-related mirror neurons in the monkey mid-posterior insula, which discharge both during execution of hand-to-mouth motor acts or observation of grooming actions (Ishida et al., 2015). In line with these findings, a recent human study showed specific vicarious responses for affective touch in the posterior portion of the insula (Morrison et al., 2011). In monkeys, it has also been shown that SII and posterior insular neurons respond to receiving pleasant touch (Grandi and Gerbella, 2016). These data suggest posterior insula might be a site for integration of observed and experienced affiliative touch (Ishida et al., 2015).

Overall, and in support of recent human fMRI data (Chan and Baker, 2015), our current monkey fMRI data show little evidence of tactile SI and SII regions in responding during observation of touch, and suggests such responses are present in parietal area PFG. Interestingly, our present study nonetheless suggests that more anterior somatosensory (visual SII) and posterior insular regions yield multimodal responses when the subject performs or observes manual actions involving touch, in agreement with 
single-cell data (Ishida et al., 2013, 2015; Hihara et al., 2015). Electrophysiological investigations will be needed to examine the detailed multimodal response characteristics of the underlying cells, and whether these apparent vicarious responses either reflect the somatosensory aspects associated with observing typical grasping and interaction movements, or represent the somatosensory state of others (Keysers et al., 2010).

\section{References}

Blakemore SJ, Bristow D, Bird G, Frith C, Ward J (2005) Somatosensory activations during the observation of touch and a case of vision-touch synaesthesia. Brain 128:1571-1583. CrossRef Medline

Bonini L, Rozzi S, Serventi FU, Simone L, Ferrari PF, Fogassi L (2010) Ventral premotor and inferior parietal cortices make distinct contribution to action organization and intention understanding. Cereb Cortex 20: 1372-1385. CrossRef Medline

Borra E, Gerbella M, Rozzi S, Luppino G (2017) The macaque lateral grasping network: a neural substrate for generating purposeful hand actions. Neurosci Biobehav Rev 75:65-90. CrossRef Medline

Bremmer F, Schlack A, Shah NJ, Zafiris O, Kubischik M, Hoffmann K, Zilles K, Fink GR (2001) Polymodal motion processing in posterior parietal and premotor cortex: a human fMRI study strongly implies equivalencies between humans and monkeys. Neuron 29:287-296. CrossRef Medline

Bufalari I, Aprile T, Avenanti A, Di Russo F, Aglioti SM (2007) Empathy for pain and touch in the human somatosensory cortex. Cereb Cortex 17: 2553-2561. CrossRef Medline

Burton H, Fabri M, Alloway K (1995) Cortical areas within the lateral sulcus connected to cutaneous representations in areas $3 \mathrm{~b}$ and 1 : a revised interpretation of the second somatosensory area in macaque monkeys. J Comp Neurol 355:539-562. CrossRef Medline

Catmur C, Walsh V, Heyes C (2007) Sensorimotor learning configures the human mirror system. Curr Biol 17:1527-1531. CrossRef Medline

Chan AW, Baker CI (2015) Seeing is not feeling: posterior parietal but not somatosensory cortex engagement during touch observation. J Neurosci 35:1468-1480. CrossRef Medline

Chen LM, Dillenburger BC, Wang F, Tang CH (2012) Differential fMRI activation to noxious heat and tactile stimuli in parasylvian areas of new world monkeys. Pain 153:158-169. CrossRef Medline

Disbrow E, Litinas E, Recanzone GH, Padberg J, Krubitzer L (2003) Cortical connections of the second somatosensory area and the parietal ventral area in macaque monkeys. J Comp Neurol 462:382-399. CrossRef Medline

Ebisch SJ, Perrucci MG, Ferretti A, Del Gratta C, Romani GL, Gallese V (2008) The sense of touch: embodied simulation in a visuotactile mirroring mechanism for observed animate or inanimate touch. J Cogn Neurosci 20:1611-1623. CrossRef Medline

Evangeliou MN, Raos V, Galletti C, Savaki HE (2009) Functional imaging of the parietal cortex during action execution and observation. Cereb Cortex 19:624-639. CrossRef Medline

Ferrari PF, Rozzi S, Fogassi L (2005) Mirror neurons responding to observation of actions made with tools in monkey ventral premotor cortex. J Cogn Neurosci 17:212-226. CrossRef Medline

Ferri S, Rizzolatti G, Orban GA (2015) The organization of the posterior parietal cortex devoted to upper limb actions: an fMRI study. Hum Brain Mapp 36:3845-3866. CrossRef Medline

Fiave PA, Jastorff J, Nelissen K (2017) Investigating shared representations of observed and executed actions using cross-modal fMRI decoding in rhesus monkeys. Society for Neuroscience. Washington, 11-15 November, Abstract No. 62.06/EE11.

Fitzgerald PJ, Lane JW, Thakur PH, Hsiao SS (2004) Receptive field properties of the macaque second somatosensory cortex: evidence for multiple functional representations. J Neurosci 24:11193-11204. CrossRef Medline

Fogassi L, Ferrari PF, Gesierich B, Rozzi S, Chersi F, Rizzolatti G (2005) Parietal lobe: from action organization to intention understanding. Science 308:662-667. CrossRef Medline

Friedman DP, Jones EG, Burton H (1980) Representation pattern in the second somatic sensory area of the monkey cerebral cortex. J Comp Neurol 192:21-41. CrossRef Medline

Friston KJ, Holmes AP, Worsley KJ, Poline JP, Frith CD, Frackowiak RS (1994) Statistical parametric maps in functional imaging: a general linear approach. Hum Brain Mapp 2:189-210. CrossRef
Gazzola V, Keysers C (2009) The observation and execution of actions share motor and somatosensory voxels in all tested subjects: single-subject analyses of unsmoothed fMRI data. Cereb Cortex 19:1239-1255. CrossRef Medline

Goda N, Yokoi I, Tachibana A, Minamimoto T, Komatsu H (2016) Crossmodal association of visual and haptic material properties of objects in the monkey ventral visual cortex. Curr Biol 26:928-934. CrossRef Medline

Grandi LC, Gerbella M (2016) Single neurons in the insular cortex of a macaque monkey respond to skin brushing: preliminary data of the possible representation of pleasant touch. Front Behav Neurosci 10: 90. CrossRef Medline

Hebart MN, Görgen K, Haynes JD (2014) The decoding toolbox (TDT): a versatile software package for multivariate analyses of functional imaging data. Front Neuroinform 8:88. CrossRef Medline

Heyes C (2010) Mesmerising mirror neurons. Neuroimage 51:789-791. CrossRef Medline

Hihara S, Taoka M, Tanaka M, Iriki A (2015) Visual responsiveness of neurons in the secondary somatosensory area and its surrounding parietal operculum regions in awake macaque monkeys. Cereb Cortex 25:45354550. CrossRef Medline

Hinkley LB, Krubitzer LA, Nagarajan SS, Disbrow EA (2007) Sensorimotor integration in S2, PV, and parietal rostroventral areas of the human sylvian fissure. J Neurophysiol 97:1288-1297. CrossRef Medline

Ishida H, Fornia L, Grandi LC, Umiltà MA, Gallese V (2013) Somato-motor haptic processing in posterior inner perisylvian region (SII/pIC) of the macaque monkey. PLoS One 8:e69931. CrossRef Medline

Ishida H, Suzuki K, Grandi LC (2015) Predictive coding accounts of shared representations in parieto-insular networks. Neuropsychologia 70:442454. CrossRef Medline

Jezzini A, Caruana F, Stoianov I, Gallese V, Rizzolatti G (2012) Functional organization of the insula and inner perisylvian regions. Proc Natl Acad Sci U S A 109:10077-10082. CrossRef Medline

Kaas JH (1993) The functional organization of somatosensory cortex in primates. Ann Anat 175:509-518. CrossRef Medline

Keysers C, Wicker B, Gazzola V, Anton JL, Fogassi L, Gallese V (2004) A touching sight: SII/PV activation during the observation and experience of touch. Neuron 42:335-346. CrossRef Medline

Keysers C, Kaas JH, Gazzola V (2010) Somatosensation in social perception. Nat Rev Neurosci 11:417-428. CrossRef Medline

Kilner JM, Lemon RN (2013) What we know currently about mirror neurons. Curr Biol 23:R1057-R1062. CrossRef Medline

Kolster H, Mandeville JB, Arsenault JT, Ekstrom LB, Wald LL, Vanduffel W (2009) Visual field map clusters in macaque extrastriate visual cortex. J Neurosci 29:7031-7039. CrossRef Medline

Krubitzer L, Clarey J, Tweedale R, Elston G, Calford M (1995) A redefinition of somatosensory areas in the lateral sulcus of macaque monkeys. J Neurosci 15:3821-3839. Medline

Kuehn E, Trampel R, Mueller K, Turner R, Schütz-Bosbach S (2013) Judging roughness by sight-A 7-tesla fMRI study on responsivity of the primary somatosensory cortex during observed touch of self and others. Hum Brain Mapp 34:1882-1895. CrossRef Medline

Mandeville JB, Marota JJ, Ayata C, Zaharchuk G, Moskowitz MA, Rosen BR, Weisskoff RM (1999) Evidence of a cerebrovascular postarteriole windkessel with delayed compliance. J Cereb Blood Flow Metab 19:679-689. CrossRef Medline

Meyer K, Kaplan JT, Essex R, Damasio H, Damasio A (2011) Seeing touch is correlated with content-specific activity in primary somatosensory cortex. Cereb Cortex 21:2113-2121. CrossRef Medline

Molenberghs P, Cunnington R, Mattingley JB (2012) Brain regions with mirror properties: a meta-analysis of 125 human fMRI studies. Neurosci Biobehav Rev 36:341-349. CrossRef Medline

Mooney R (2014) Auditory-vocal mirroring in songbirds. Philos Trans R Soc Lond B Biol Sci 369:20130179. CrossRef Medline

Morrison I, Björnsdotter M, Olausson H (2011) Vicarious responses to social touch in posterior insular cortex are tuned to pleasant caressing speeds. J Neurosci 31:9554-9562. CrossRef Medline

Nelissen K, Luppino G, Vanduffel W, Rizzolatti G, Orban GA (2005) Observing others: multiple action representation in the frontal lobe. Science 310:332-336. CrossRef Medline

Nelissen K, Vanduffel W (2011) Grasping-related functional magnetic resonance imaging brain responses in the macaque monkey. J Neurosci 31: 8220-8229. CrossRef Medline 
Nelissen K, Borra E, Gerbella M, Rozzi S, Luppino G, Vanduffel W, Rizzolatti G, Orban GA (2011) Action observation circuits in the macaque monkey cortex. J Neurosci 31:3743-3756. CrossRef Medline

Nelissen K, Fiave PA, Vanduffel W (2018) Decoding grasping movements from the parieto-frontal reaching circuit in the nonhuman primate. Cereb Cortex 28:1245-1259. CrossRef Medline

Oosterhof NN, Tipper SP, Downing PE (2013) Crossmodal and action-specific: neuroimaging the human mirror neuron system. Trends Cogn Sci 17:311-318. CrossRef Medline

Pihko E, Nangini C, Jousmäki V, Hari R (2010) Observing touch activates human primary somatosensory cortex. Eur J Neurosci 31:1836-1843. CrossRef Medline

Pons TP, Garraghty PE, Mishkin M (1988) Lesion-induced plasticity in the second somatosensory cortex of adult macaques. Proc Natl Acad Sci U S A 85:5279-5281. CrossRef Medline

Press C, Catmur C, Cook R, Widmann H, Heyes C, Bird G (2012) fMRI evidence of "mirror" responses to geometric shapes. PLoS One 7:e51934. CrossRef Medline

Pulvermüller F, Fadiga L (2010) Active perception: sensorimotor circuits as a cortical basis for language. Nat Rev Neurosci 11:351-360. CrossRef Medline

Raos V, Savaki HE (2016) Perception of actions performed by external agents presupposes knowledge about the relationship between action and effect. Neuroimage 132:261-273. CrossRef Medline

Raos V, Evangeliou MN, Savaki HE (2004) Observation of action: grasping with the mind's hand. Neuroimage 23:193-201. CrossRef Medline

Rizzolatti G, Sinigaglia C (2016) The mirror mechanism: a basic principle of brain function. Nat Rev Neurosci 17:757-765. CrossRef Medline

Robinson CJ, Burton H (1980) Somatic submodality distribution within the second somatosensory (SII), 7b, retroinsular, postauditory, and granular insular cortical areas of M. fascicularis. J Comp Neurol 192:93-108. CrossRef Medline

Rossetti A, Miniussi C, Maravita A, Bolognini N (2012) Visual perception of bodily interactions in the primary somatosensory cortex. Eur J Neurosci 36:2317-2323. CrossRef Medline
Rozzi S, Coudé G (2015) Grasping actions and social interaction: neural bases and anatomical circuitry in the monkey. Front Psychol 6:973. CrossRef Medline

Rozzi S, Ferrari PF, Bonini L, Rizzolatti G, Fogassi L (2008) Functional organization of inferior parietal lobule convexity in the macaque monkey: electrophysiological characterization of motor, sensory and mirror responses and their correlation with cytoarchitectonic areas. Eur J Neurosci 28:1569-1588. CrossRef Medline

Schaefer M, Xu B, Flor H, Cohen LG (2009) Effects of different viewing perspectives on somatosensory activations during observation of touch. Hum Brain Mapp 30:2722-2730. CrossRef Medline

Schneider RJ, Friedman DP, Mishkin M (1993) A modality-specific somatosensory area within the insula of the rhesus monkey. Brain Res 621: 116-120. CrossRef Medline

Shokur S, O’Doherty JE, Winans JA, Bleuler H, Lebedev MA, Nicolelis MA (2013) Expanding the primate body schema in sensorimotor cortex by virtual touches of an avatar. Proc Natl Acad Sci U S A 110:15121-15126. CrossRef Medline

Vanduffel W, Fize D, Mandeville JB, Nelissen K, Van Hecke P, Rosen BR, Tootell RB, Orban GA (2001) Visual motion processing investigated using contrast agent-enhanced fMRI in awake behaving monkeys. Neuron 32:565-577. CrossRef Medline

Wu R, Wang F, Yang PF, Chen LM (2017) High-resolution functional MRI identified distinct global intrinsic functional networks of nociceptive posterior insula and S2 regions in squirrel monkey brain. Neuroimage 155: 147-158. CrossRef Medline

Zhao F, Wang P, Hendrich K, Ugurbil K, Kim SG (2006) Cortical layerdependent BOLD and CBV responses measured by spin-echo and gradient-echo fMRI: insights into hemodynamic regulation. Neuroimage 30:1149-1160. CrossRef Medline

Zhou YD, Fuster JM (2000) Visuo-tactile cross-modal associations in cortical somatosensory cells. Proc Natl Acad Sci U S A 97:9777-9782. CrossRef Medline 